ESAIM: PROCEEDINGS, November 2005, Vol.15, 87-120

T. Goudon, E. Sonnendrucker \& D. Talay, Editors

DOI: $10.1051 /$ proc:2005024

\title{
HYBRID MULTISCALE METHODS FOR HYPERBOLIC AND KINETIC PROBLEMS *
}

\author{
LORENZO PARESCHI ${ }^{1}$
}

\begin{abstract}
In these notes we present some recent results on the development of hybrid methods for hyperbolic and kinetic equations with multiple scales. The main ingredients in the schemes are a suitable merging of particle methods in non stiff regimes with high resolution shock capturing techniques in stiff ones. The key aspect in the development of the algorithms is the choice of a suitable hybrid representation of the solution.

First, after a brief review on sampling methods and Monte Carlo techniques, we introduce the hybrid schemes for hyperbolic systems with relaxation and present numerical applications to the simple JinXin relaxation model both in one and two space dimensions. Next, we show how to extend the above methodology to the case of kinetic models of BGK type and discuss the challenging case of the full Boltzmann equation. Some numerical results are also presented.
\end{abstract}

Résumé. Nous présentons dans ces notes quelques résultats récents concernant le développement de méthodes hybrides pour les équations hyperboliques et cinétiques. L'ingrédient principal de ces schémas réside dans un mélange pertinent de méthodes particulaires dans les régimes réguliers et de techniques haute résolution de capture de choc dans les zones raides. La clef du développement de ces algorithmes est donnée par le choix d'une bonne représentation hybride de la solution.

Après une brève revue des méthodes d'échantillonnage et des techniques Monte-Carlo, nous introduisons des schémas hybrides pour des systèmes hyperboliques avec relaxation et présentons des applications numériques pour le modèle de Jin-Xin, en dimension un et deux. Ensuite, nous montrons comment cette méthodologie s'étend à des modèles cinétiques de type BGK et discutons le cas délicat de l'équation de Boltzmann. Des résultats numériques sont aussi présentés pour ces situations.

Keywords: multiscale problems, particle methods, high-resolution schemes, hyperbolic conservation laws, stiff terms, kinetic equations

AMS Subject classification: 65M99, 65L06, 82D05

\section{Contents}

1. Introduction

2. Hyperbolic relaxation systems

3. Monte Carlo and sampling techniques $\quad 90$

3.1. Inverse transform methods 90

3.2. Acceptance-rejection methods $\quad 91$

* This work was partially supported by the European network HYKE, funded by the EC as contract HPRN-CT-2002-00282 and by the project NUMSTAT funded by the University of Ferrara.

${ }^{1}$ Department of Mathematics, University of Ferrara, Via Machiavelli 35, 44100 Ferrara Italy. E-mail: pareschi@dm.unife.it. 
3.3. Discrete sampling methods $\quad 91$

3.4. Stratified sampling methods 92

3.5. Reconstruction methods $\quad 94$

4. Hybrid methods $\quad 95$

4.1. Monte Carlo methods (MCM) 96

4.2. The hybrid method (HM) 98

4.3. A Componentwise hybrid method (CHM) 100

5. Implementation and numerical tests 102

5.1. Solving the equilibrium part 102

5.2. Numerical examples 103

$\begin{array}{lr}\text { 6. Kinetic equations } & 104\end{array}$

6.1. BGK models $r$

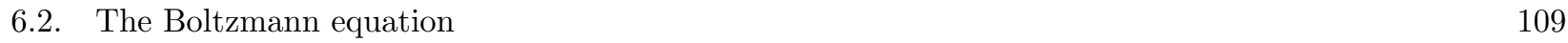

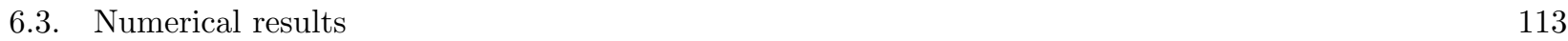

$\begin{array}{ll}\text { 7. Concluding Remarks } & 114\end{array}$

References
R

\section{INTRODUCTION}

Many problems of applied sciences represent a challenge for numerical methods due to the presence of different time and/or space scales (for example mesoscopic and microscopic modelling of transport problems [13,14], wave propagation in presence of sources [16,44,70], mathematical finance [7], biomathematics [15,61], computer graphics $[6], \ldots)$. Furthermore, the presence of additional model dependent multiscale parameters contribute to increase the computational difficulties associated with such problems. In these systems, besides conventional deterministic discretizations, a probabilistic approach is highly desirable.

Monte Carlo methods or probabilistic techniques at different levels are widely used to simulate complex systems $[8,30,37,39,43]$. They have many advantages in terms of computational cost for problems with high dimensions, simplicity in preserving some physical properties of the underlying problem (typically using a particle interpretation of the statistical sample) and great flexibility when dealing with complicate geometries.

In many applications, for example in stiff relaxation-like systems and in kinetic equations, we often have a natural strong dimension reduction of the model due to a the large variation of some parameters [16,36,44,49]. Domain decomposition techniques are then used in order to better adapt the modeling strategy and the design of the numerical schemes. However this multi-modelling approach, which at the mathematical level is a consequence of asymptotic approximations, requires the a-priori knowledge of some of the scales in the problem which are typically hard to know in practice $[4,40,67]$.

A complementary strategy would be to use the full model in the whole computational domain and to design the numerical method in such a way that it is capable to take advantage of the model reduction induced by the presence of small scales $[9,31-35,47,50]$. Off course this would involve the development of heterogeneous numerical methods which hybridize different numerical approaches of probabilistic and deterministic nature.

The most classical example is perhaps the case of the Boltzmann equation which, due to the high dimensionality and to the necessity to avoid artificial truncation in the velocity field, is solved by Monte Carlo schemes $[2,3,48,55]$. However there are important flow regimes where Monte Carlo schemes loose his effectiveness: flow for which the Knudsen number is small enough that the collision rate is large and the flow is well described by fluid mechanics. In such cases a conventional deterministic Navier-Stokes solver would certainly be more appropriate.

Often, the design of such hybrid methodology involves not only the use hybrid numerical methods but also their efficient coupling with suitable multi-modelling strategies. Clearly the details of the schemes are rather 
problem dependent $[1,10,18,20,29,38,42,51,52,62,63,65]$. We quote the recent works by Weinan E and Bjorn Engquist for a general approach to heterogeneous multiscale methods in scientific computing [22-24].

In this work we describe a methodology that can be applied to design new hybrid methods for the numerical solution of a wide class of hyperbolic and kinetic problems that involve different scales. The main components of the schemes is the use of probabilistic Monte Carlo methods for the full model (far from equilibrium regimes) combined with deterministic shock capturing techniques for the reduced one (close to equilibrium regimes). An essential aspect in the development of the algorithms is the choice of a suitable hybrid representation of the solution $[20,51,52]$.

The rest of the notes are organized as follows. First we introduce the model problems we are considering. After a brief review of some basic tools from sampling theory we present the different schemes in the case of Jin-Xin relaxation system. Next we discuss how to extend the methods to kinetic equations with BGK and Boltzmann collision operators.

\section{HyPERBOLIC RELAXATION SYSTEMS}

We will consider here one-dimensional hyperbolic systems with relaxation of the form [16]

$$
\partial_{t} U+\partial_{x} F(U)=\frac{1}{\varepsilon} R(U), \quad x \in \mathbb{R}
$$

where $U=U(x, t) \in \mathbb{R}^{N}, F: \mathbb{R}^{N} \rightarrow \mathbb{R}^{N}$, the Jacobian matrix $F^{\prime}(U)$ has real eigenvalues and $\varepsilon>0$ is the relaxation time.

The operator $R: \mathbb{R}^{N} \rightarrow \mathbb{R}^{N}$ is said a relaxation operator, and consequently (1) defines a relaxation system in the sense of Whitham and Liu [44,70], if there exists a constant $n \times N$ matrix $Q$ with $\operatorname{rank}(Q)=n<N$ such that

$$
Q R(U)=0 \quad \forall U \in \mathbb{R}^{N} .
$$

This gives $n$ independent conserved quantities $v=Q U$. Moreover such conserved quantities uniquely determine a local equilibrium value

$$
U=\mathcal{E}(v) \text { such that } R(\mathcal{E}(v))=0 \text {. }
$$

The image of $\mathcal{E}$ represents the manifold of local equilibria of the relaxation operator $R$. Using (2) in (1) we obtain a system of $n$ conservation laws which is satisfied by every solution of (1)

$$
\partial_{t}(Q U)+\partial_{x}(Q F(U))=0
$$

For small values of the relaxation parameter $\varepsilon$ from (1) we get $R(U)=0$ which by (3) implies $U=\mathcal{E}(v)$. In this case system (1) is well approximated by the reduced system

$$
\partial_{t} v+\partial_{x} G(v)=0
$$

where $G(v)=Q F(\mathcal{E}(v))$.

Let us recall that several physical phenomena of great importance for applications are described by hyperbolic systems with relaxation terms, for example kinetic models with discrete velocities and hydrodynamical models with sources. We refer to $[16,44,70]$ for a theoretical study of these relaxation problems.

A simple prototype example of relaxation system in the case $N=2$ is given by the $p$-system

$$
\begin{aligned}
& \partial_{t} u+\partial_{x} v=0, \\
& \partial_{t} v+\partial_{x} p(u)=-\frac{1}{\varepsilon}(v-f(u)),
\end{aligned}
$$

which corresponds to take $U=(u, v), F(U)=(v, p(u)), R(U)=(0, f(u)-v)$. 
For small values of $\varepsilon$ from the second equation in (6) we get the local equilibrium

$$
v=f(u)
$$

and under Liu's subcharacteristic condition $p^{\prime}(u)>f^{\prime}(u)^{2}$ solutions to $(6)$ converges to the solution of the scalar conservation law

$$
\partial_{t} u+\partial_{x} f(u)=0
$$

\section{Monte Carlo and sampling techniques}

In this section give a brief review of some classical random sampling techniques [30,37, 43]. In particular we will focus on variance reduction techniques based on the use of piecewise polynomial approximations. For simplicity we describe the methods only for probability densities of a single random variable.

\subsection{Inverse transform methods}

We start by recalling the main result which is at the basis of inverse transform methods.

Theorem 1. Let $X$ be a random variable with density $p_{X}(x), x \in \mathbb{R}$, i.e. $p_{X}(x) \geq 0, \int_{\mathbb{R}} p_{X}(x) d x=1$, then its cumulative random variable

$$
C(X)=\int_{-\infty}^{X} p_{X}(x) d x
$$

is uniformly distributed in $[0,1]$.

The above theorem states that, independently from $p_{X}(x)$, the cumulative random variable $C(X)$ is always uniform $[0,1]$.

Let us recall that given a random variable $X$ with density $p_{X}(x)$ its corresponding distribution function is defined as

$$
P_{X}(x)=\int_{-\infty}^{x} p_{X}(y) d y .
$$

As a consequence we have $C=P_{X}(X)$, and thus $X=P_{X}^{-1}(C)$ where $C$ is uniform $[0,1]$. Then the random variable $X$ can be sampled by extracting a uniformly distributed random number $\xi$, and then taking

$$
x=P_{X}^{-1}(\xi) .
$$

Example 3.1. Let $p_{X}(x)=\exp (-x), x \geq 0$. Then

$$
P_{X}(x)=\int_{0}^{x} \exp (-y) d y=1-\exp (-x)=\xi,
$$

and therefore

$$
x=-\ln (1-\xi)
$$

or $x=-\ln \xi$, because $1-\xi$ is also a uniformly distributed $[0,1]$ random number.

The applicability of the inverse transform method can be extended to the case in which the primitive of $p_{X}(x)$ is not easy to invert. In this case one can solve numerically, with a suitable iterative method, the equation

$$
P_{X}(x)=\xi
$$

for a given uniform random number $\xi$ in $[0,1]$. Off course considerations about the increase in computational cost of the method should be done accordingly to the particular structure of the primitive. An alternative approach is explained in the next paragraph. 


\subsection{Acceptance-rejection methods}

In practical applications it may be expensive to use the inverse transform method, since in general a nonlinear equation has to be solved. In such cases a different technique, called acceptance-rejection, can be used to sample a random variables with a given density.

Let $X$ be a random variable with density $p_{X}(x)$. Then we look for a function $w(x) \geq p_{X}(x) \forall x \in \mathbb{R}$ whose primitive $W(x)$ is easily invertible. Let $A=\int_{-\infty}^{\infty} w(x) d x$. Then the algorithm works as follows

Algorithm 3.2 (Acceptance-rejection sampling).

(1) sample $x$ from $w(x) / A$ using the equation $x=W^{-1}\left(A \xi_{1}\right)$, where $\xi_{1}$ is a uniformly distributed $[0,1]$ random number

(2) extract a second uniform $[0,1]$ random number $\xi_{2}$ and generate a uniformly distributed $[0, w(x)]$ random number $y$ setting $y=\xi_{2} w(x)$

(3) if $y<p_{X}(x)$ then accept the sample, else reject the sample and repeat step 1.

It is clear that the efficiency of the scheme depends on how easy it is to invert the function $W(x)$ and how frequently we accept the sample. The fraction of accepted samples equals the ratio of the areas below the two curves $p_{X}(x)$ and $W(x)$ and it is therefore equal to $1 / A$.

If the function $p_{X}(x)$ ca be factorized as $p_{X}(x)=C g(x) w(x)$ where $C \geq 1$ is a constant, the primitive $W(x)$ of $w(x)$ is easily invertible and $0 \leq g(x) \leq 1$ is the weight function, we can apply a weighted acceptance-rejection strategy as follows

Algorithm 3.3 (Weighted acceptance-rejection sampling).

(1) sample $x$ from $w(x) / A$ using the equation $x=W^{-1}\left(A \xi_{1}\right)$, where $\xi_{1}$ is a uniformly distributed $[0,1]$ random number

(2) extract a second uniform $[0,1]$ random number $\xi_{2}$

(3) if $\xi_{2}<g(x)$ then accept the sample, else reject the sample and repeat step 1.

Note that taking $g(x)=p_{X}(x) / w(x)$ (here $C=1$ ) we have the usual acceptance rejection method.

\subsection{Discrete sampling methods}

Sometimes a density function is given as a convex combination of simpler density functions,

$$
p(x)=\sum_{i=1}^{M} w_{i} p_{i}(x)
$$

where $w_{i}$ are probabilities (i.e. $w_{i} \geq 0, \sum_{i=1}^{M} w_{i}=1$ ), and $p_{i}(x)$ are probability densities. In that case the sampling can be performed as follows

Algorithm 3.4 (Sampling from a convex combination).

(1) select an integer $i \in\{1, \ldots, M\}$ with probability $w_{i}$

(2) sample $x$ from a random variable with density $p_{i}(x)$.

Because of the relevance in several applications, step 1 of the previous algorithm deserves an extended discussion.

Let us suppose that $k \in\{1, \ldots, M\}$ is an integer random number, with probabilities $\left\{w_{k}\right\}$. In order to sample $k$ with probability $w_{k}$ we can divide interval $[0,1]$ in $M$ intervals, $i$-th interval being of length $w_{i}$, extract a uniform $[0,1]$ random number $\xi$, and detect the interval $k$ to which $\xi$ belongs.

This can be done in the following way 
Algorithm 3.5 (Discrete sampling).

(1) compute $W_{k}=\sum_{i=1}^{k} w_{k}, k=1, \ldots, M, \quad W_{0}=0$

(2) extract a uniform $[0,1]$ random number $\xi$

(3) find the integer $k$ such that $W_{k-1} \leq \xi<W_{k}$.

For an arbitrary set of probabilities $\left\{w_{i}\right\}$, once $W_{i}$ have been computed, step 3 can be performed with a binary search, in $O(\ln M)$ operations.

This approach is efficient if the numbers $W_{k}$ can be computed once, and then used several times, and if $M$ is not too large. In several circumstances, however, the number $M$ may be very large, and the numbers $w_{k}$ may change, or, in other words, one is interested in sampling $k$ with probability $w_{k}$ without explicitly computing all the probabilities $w_{k}$. This can be done by using the acceptance-rejection technique, if at least an estimate $\bar{w}$ such that $\bar{w} \geq w_{i}, i=1, \ldots, M$ is known. The procedure is used as follows

Algorithm 3.6 (Discrete sampling with upper bound $\bar{w}$ ).

(1) select an integer random number uniformly in $[1, \ldots, M]$, as

$$
k=\llbracket M \xi_{1} \rrbracket+1
$$

where $\llbracket x \rrbracket$ denotes the integer part of $x$,

(2) if $w_{k} \xi_{2}<\bar{w}$ then accept the sampling else reject it and repeat point 1.

Clearly the procedure can be generalized to the case in which the estimate depends on $k$, i.e. $\bar{w}_{k} \geq w_{k}, k=$ $1, \ldots, M$.

\subsection{Stratified sampling methods}

One of the main drawback of the acceptance-rejection techniques described above is the large variance of the samples we obtain. This is mainly due to the fact that we sample from the whole interval of interest of the distribution function. The basic principle of stratified sampling is to divide the sampling interval up into subintervals. You then perform an inverse transform sampling or an acceptance-rejection approach on each subinterval. The reason you might use this method is that now instead of finding the variance in one big go, you can find the variance by adding up the variances of each subinterval. Note also that this approach is rather natural in a method that couples a deterministic grid based strategy with a Monte Carlo approach. In fact the numerical solution is typically represented by a piecewise polynomial approximation on a grid so that we can easily perform a direct sampling in each subinterval.

We describe the method for some typical piecewise polynomial approximations that are used in finite difference and finite volumes methods for hyperbolic conservation laws. To this aim we introduce the grid points

$$
x_{j}=j \Delta x, \quad x_{j+1 / 2}=x_{j}+\frac{1}{2} \Delta x, \quad j=\ldots,-2,-1,0,1,2, \ldots
$$

and will use the standard notation for the cell averages of the function $u(x), x \in \mathbb{R}$

$$
u_{j+1 / 2}=\frac{1}{\Delta x} \int_{I_{j}} u(x) d x
$$

where $I_{j}=\left[x_{j}, x_{j+1}\right]$, and for the point values of the function $u_{j}=u\left(x_{j}\right)$. In the sequel we assume that

$$
A=\int_{\mathbb{R}} u(x) d x
$$

is finite. 
Starting from cell averages the corresponding piecewise constant approximation $u_{P C}(x)$ of $u(x)$ is second order accurate. An algorithm to take $N$ samples $p_{i}$ from $u_{P C}(x)$ is the following

Algorithm 3.7 (Piecewise constant sampling).

(1) set $k=0$ and $m=\frac{\Delta x}{N} \sum_{j} u_{j+1 / 2}$

(2) for each interval $I_{j}, j=\ldots,-2,-1,0,1,2, \ldots$

(a) set $N_{j}=$ Iround $\left(\frac{\Delta x}{m} u_{j+1 / 2}\right)$

(b) for $i=1, \ldots, N_{j}$ extract a uniform $[0,1]$ random number $\xi$ set $d x=\frac{(1-2 \xi) \Delta x}{2}$ and set $p_{k+i}=x_{j+1 / 2}+d x$ set $k=k+N_{j}$

Here by $\operatorname{Iround}(x)$ we denote a suitable integer rounding of a positive real number $x$. In our algorithm we choose

$$
\operatorname{Iround}(x)=\left\{\begin{array}{lll}
\llbracket x \rrbracket+1 & \text { with probability } & x-\llbracket x \rrbracket \\
\llbracket x \rrbracket & \text { with probability } & \llbracket x \rrbracket+1-x
\end{array}\right.
$$

Similarly from pointwise values we consider the piecewise linear approximation $u_{P L}(x)$ of $u(x)$ which is also second order accurate. In this case, given a uniform random variable $\xi$ in the interval $[0,1]$, in each interval $\left[x_{j}, x_{j+1}\right]$ we have to invert relation

$$
P_{j}(x):=\frac{1}{A_{j}}\left(x-x_{j}\right)\left(\left(u_{j+1}-u_{j}\right)\left(x-x_{j}\right)+2 u_{j}\right)=\xi,
$$

where $A_{j}=\left(u_{j}+u_{j+1}\right) / 2$. This originates the following algorithm

Algorithm 3.8 (Piecewise linear sampling).

(1) set $k=0$ and $m=\frac{\Delta x}{N} \sum_{j} u_{j}$

(2) for each interval $I_{j}, j=\ldots,-2,-1,0,1,2, \ldots$

(a) set $A_{j}=\frac{1}{2}\left(u_{j}+u_{j+1}\right)$ and set $N_{j}=\operatorname{Iround}\left(\frac{\Delta x}{m} A_{j}\right)$

(b) for $i=1, \ldots, N_{j}$ extract a uniform $[0,1]$ random number $\xi$

$$
\begin{aligned}
& \text { if } u_{j}<u_{j+1} \text { set } d x=\Delta x\left(\frac{u_{j}-\sqrt{u_{j}^{2}+2 \xi A_{j}\left(u_{j+1}-u_{j}\right)}}{u_{j}-u_{j+1}}\right) \\
& \text { else if } u_{j}>u_{j+1} \text { set } d x=\Delta x\left(1-\frac{u_{j+1}-\sqrt{u_{j+1}^{2}+2 \xi A_{j}\left(u_{j}-u_{j+1}\right)}}{u_{j+1}-u_{j}}\right) \\
& \quad \text { else set } d x=\Delta x \xi \\
& \text { set } p_{k+i}=x_{j}+d x \\
& \text { set } k=k+N_{j}
\end{aligned}
$$

Additional sampling techniques can be derived using inverse interpolation strategies. In fact the distribution function $P_{X}(x)$ of the probability density $p_{X}(x)$ is by construction monotonically increasing. For example if we introduce the discrete distribution function as

$$
P_{i}=\frac{1}{A} \int_{-\infty}^{x_{i}} u(x) d x, \quad i=\ldots,-2,-1,0,1,2, \ldots
$$


given a uniform random number $\xi$ we can find the integer $j$ such that

$$
P_{j}<\xi \leq P_{j+1}
$$

and then, using linear interpolation, compute the interpolated sample

$$
x=x_{j}+\frac{\xi-P_{j}}{P_{j+1}-P_{j}}\left(x_{j+1}-x_{j}\right) .
$$

In summary to take $N$ samples we must follow the procedure below.

Algorithm 3.9 (Sampling by inverse interpolation).

(1) compute $P_{i}=\frac{1}{A} \int_{-\infty}^{x_{i}} u(x) d x, \quad i=\ldots,-2,-1,0,1,2, \ldots$

(2) for $k=1, \ldots, N$
(a) extract a uniform $[0,1]$ random number $\xi$
(b) find the integer $j$ such that $P_{j}<\xi \leq P_{j+1}$ by binary search
(c) set $p_{k}=x_{j}+\frac{\xi-P_{j}}{P_{j+1}-P_{j}}\left(x_{j+1}-x_{j}\right)$.

\subsection{Reconstruction methods}

In this last introductive paragraph we describe briefly some reconstruction techniques. The main target here is to reconstruct the probability density $p(x)$ starting from the knowledge of a set of samples. There are many statistical techniques designed for this purpose accordingly to the field of application. Here we recall some simple methods which are particularly suitable when we are interested in reconstructing the probability density on a given regular grid $x_{j}=j \Delta x, j=\ldots,-2,-1,0,1,2, \ldots$

The simplest method, which produces a piecewise constant reconstruction, is based on evaluating the histogram of the samples on the grid. Given a set of $N$ samples $p_{1}, p_{2}, \ldots, p_{N}$ we define the discrete probability density at the cell centers

$$
p\left(x_{j+1 / 2}\right)=\frac{1}{N} \sum_{k=1}^{N} \Psi_{\Delta x}\left(p_{k}-x_{j+1 / 2}\right), \quad j=\ldots,-2,-1,0,1,2, \ldots
$$

where $\Psi_{\Delta x}(x)=1 / \Delta x$ if $|x| \leq \Delta x / 2$ and $\Psi_{\Delta x}(x)=0$ elsewhere.

Smoother reconstructions can be recovered by convolving the samples with a suitable mollifier. More precisely

$$
p\left(x_{j+1 / 2}\right)=\frac{1}{N} \sum_{k=1}^{N} W_{\Delta x}\left(p_{k}-x_{j+1 / 2}\right), \quad j=\ldots,-2,-1,0,1,2, \ldots
$$

where $W_{\Delta x} \geq 0$ is a smoothed approximation of $\Psi_{\Delta x}$ such that

$$
\Delta x \int_{\mathbb{R}} W_{\Delta x}(x) d x=1 .
$$

For example, we can take $W_{\Delta x}$ as a B-spline. We give here the explicit expression in the case of the quadratic B-spline on $[-1.5,1.5]$

$$
W_{\Delta x}(x)=\frac{1}{\Delta x} B_{2}(x)\left(\frac{x}{\Delta x}\right), \quad B_{2}(x)=\left\{\begin{array}{cl}
3 / 4-|x|^{2} & \text { if }|x| \leq 0.5 \\
(|x|-3 / 2)^{2} / 2 & \text { if } 0.5<|x| \leq 1.5 \\
0 & \text { otherwise }
\end{array}\right.
$$




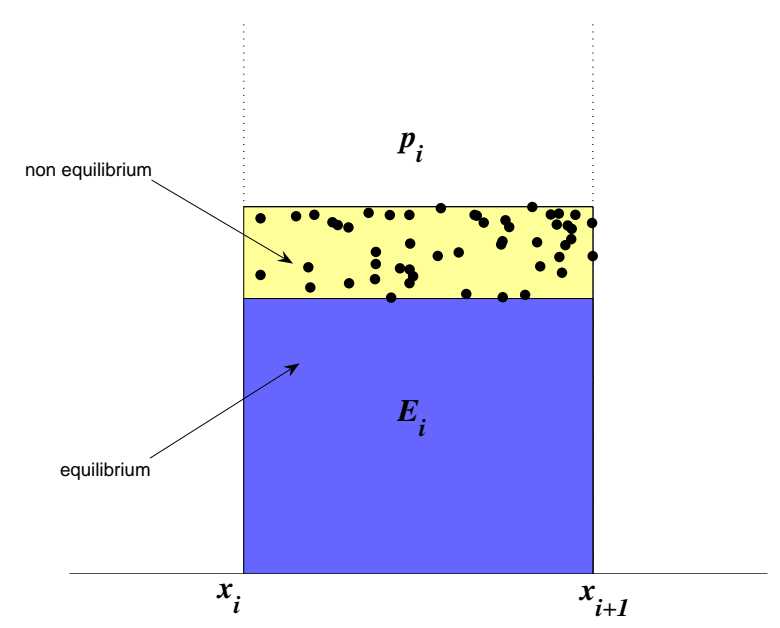

Figure 1. Hybrid representation of a cell value.

Note that the value $\Delta x$ has a strong influence on the fluctuations in the reconstructed function, and in general should be selected as a good compromise between fluctuations and resolution. Clearly the above reconstruction strategy can be used also to reconstruct the values of the probability density at the cell points $x_{i}$.

\section{Hybrid methods}

The starting point in the construction of the methods is the following definition of hybrid representation of a discrete probability density.

Definition 1. Given a discrete probability density $p_{i}, i=1, \ldots, N$ (i.e. $p_{i} \geq 0, \sum_{i} p_{i}=1$ ) and a discrete probability density $E_{i}, i=1, \ldots, N$ called equilibrium density, we define $w_{i} \in[0,1]$ and $\tilde{p}_{i} \geq 0$ in the following way

$$
w_{i}= \begin{cases}\frac{p_{i}}{E_{i}}, & p_{i} \leq E_{i} \neq 0 \\ 1, & p_{i} \geq E_{i}\end{cases}
$$

and

Thus $p_{i}$ can be represented as

$$
\tilde{p}_{i}=p_{i}-w_{i} E_{i}
$$

$$
p_{i}=\tilde{p}_{i}+w_{i} E_{i} .
$$

Remark 1. If we take

$$
\beta=\min _{i}\left\{w_{i}\right\}
$$

and

we have

$$
\tilde{p}_{i}=p_{i}-\beta E_{i},
$$

$$
\sum_{i} \tilde{p}_{i}=1-\beta .
$$

Let us define for $\beta \neq 1$ the discrete probability density

$$
p_{i}^{p}=\frac{\tilde{p}_{i}}{1-\beta} .
$$


The case $\beta=1$ is trivial since it implies $p_{i}=E_{i}, i=1, \ldots, N$. Thus the discrete probability density $p_{i}$, $i=1, \ldots, N$ can be written as a convex combination of two probability densities in the form [20,51,52]

$$
p_{i}=(1-\beta) p_{i}^{p}+\beta E_{i}
$$

Clearly the above representation is a particular case of (18).

In the case of an hyperbolic system with relaxation we recall that $U(x, t) \in \mathbb{R}^{N}$ denotes the solution of the system whereas $\mathcal{E}(v(x, t)) \in \mathbb{R}^{N}$ denotes the equilibrium state where $v(x, t) \in \mathbb{R}^{n}$ are the conserved variables.

Thus we consider the following general representation

$$
U(x, t)=\underbrace{\tilde{U}(x, t)}_{\text {nonequilibrium }}+\underbrace{W(x, t) \mathcal{E}(v(x, t))}_{\text {equilibrium }},
$$

where $W(x, t)=\operatorname{diag}\left(w_{1}(x, t), w_{2}(x, t), \ldots, w_{N}(x, t)\right), 0 \leq w_{i}(x, t) \leq 1$ is a $N \times N$ matrix that characterizes the equilibrium fraction and $\tilde{U}(x, t)$ the non equilibrium part of the solution.

The general methodology consist in

- Solve the evolution of the non equilibrium part by Monte Carlo methods. Thus $\tilde{U}(x, t)$ will be represented by a set of samples in the computational domain.

- Solve the evolution of the equilibrium part by deterministic methods. Thus $W(x, t) \mathcal{E}(v(x, t))$ will be represented on a suitable grid in the computational domain.

In the sequel, we will describe the different schemes in the case of Jin-Xin relaxation system.

$$
\begin{aligned}
\partial_{t} u+\partial_{x} v & =0 \\
\partial_{t} v+a \partial_{x} u & =-\frac{1}{\varepsilon}(v-F(u)) .
\end{aligned}
$$

As $\varepsilon \rightarrow 0$, under the subcharacteristic condition $a>f^{\prime}(u)^{2}$ we obtain the scalar conservation law $u_{t}+\partial_{x} F(u)=$ 0 .

In order to introduce the reader to the main tools used we start the section by describing a simple Monte Carlo approach where the entire solution is represented by samples [56].

\subsection{Monte Carlo methods (MCM)}

First we rewrite the system in diagonal form

$$
\begin{gathered}
\partial_{t} f+\sqrt{a} \partial_{x} f=-\frac{1}{\varepsilon}\left(f-E_{f}(u)\right) \\
\partial_{t} g-\sqrt{a} \partial_{x} g=-\frac{1}{\varepsilon}\left(g-E_{g}(u)\right) . \\
f=\frac{\sqrt{a} u+v}{2 \sqrt{a}}, \quad g=\frac{\sqrt{a} u-v}{2 \sqrt{a}}, \quad E_{f}(u)=\frac{\sqrt{a} u+F(u)}{2 \sqrt{a}}, \quad E_{g}(u)=\frac{\sqrt{a} u-F(u)}{2 \sqrt{a}} .
\end{gathered}
$$

We assume $-u \sqrt{a} \leq F(u) \leq u \sqrt{a}$ and $u \geq 0$ so that $f, g \geq 0$. This is guaranteed by the subcharacteristic condition if $F(0)=\overline{0}$.

We start by splitting the system in the two separate steps, a relaxation step represented by a system of stiff ordinary differential equations

$$
\begin{aligned}
\partial_{t} f^{r} & =-\frac{1}{\varepsilon}\left(f^{r}-E_{f}\left(u^{r}\right)\right) \\
\partial_{t} g^{r} & =-\frac{1}{\varepsilon}\left(g^{r}-E_{g}\left(u^{r}\right)\right)
\end{aligned}
$$


and a convection step

$$
\begin{aligned}
\partial_{t} f^{c}+\sqrt{a} \partial_{x} f^{c} & =0 \\
\partial_{t} g^{c}-\sqrt{a} \partial_{x} g^{c} & =0
\end{aligned}
$$

Note that, given an initial data $f(x, 0)$ and $g(x, 0)$, we can easily compute the exact solution of the relaxation step as

$$
\begin{aligned}
& f^{r}(x, t)=e^{-t / \varepsilon} f(x, 0)+\left(1-e^{-t / \varepsilon}\right) E_{f}(u(x, 0)), \\
& g^{r}(x, t)=e^{-t / \varepsilon} g(x, 0)+\left(1-e^{-t / \varepsilon}\right) E_{g}(u(x, 0)),
\end{aligned}
$$

this solution is then used as initial data for the transport step to get the approximate solution at time $t$. We recall that $u^{r}(x, t)=u(x, 0)$ during the relaxation step.

In the case of nonnegative initial data and if $E_{f}, E_{g} \geq 0$, the solution of our problem can be sought in the form of a discrete probability density at each space point

$$
p(x, v, t)= \begin{cases}\frac{f(x, t)}{u(x, t)}, & v=\sqrt{a}, \\ \frac{g(x, t)}{u(x, t)}, & v=-\sqrt{a} .\end{cases}
$$

Let us define with $\left\{\nu_{1}, \nu_{2}, \ldots, \nu_{N}\right\}$ the initial samples from $p(x, v, 0)$ at a given space point $x$, we know that $\nu_{k}= \pm \sqrt{a}, k=1, \ldots, N$. Hence a Monte Carlo method to obtain samples from $p^{r}(x, v, t)$ with $f^{r}(x, t)$ and $g^{r}(x, t)$ solutions of the relaxation step is

Algorithm 4.1 (Simple Monte Carlo for Jin-Xin relaxation).

(1) Given a sample $\nu_{k}$

(a) with probability $e^{-t / \varepsilon}$ the sample is unchanged

(b) with probability $1-e^{-t / \varepsilon}$ the sample is replaced with an equilibrium sample. To extract an equilibrium sample proceed as follows

(i) with probability $\frac{E_{f}(u(x, 0))}{u(x, 0)}$ take $\nu_{k}=\sqrt{a}$

(ii) with probability $\frac{E_{g}(u(x, 0))}{u(x, 0)}$ take $\nu_{k}=-\sqrt{a}$.

Note that the above procedure requires the exact knowledge of $u(x, 0)$ which we can only estimate from the samples at the given point $x$.

In practice we can integrate equations (24-25) over the cell $I_{j}$ and write, up to second order accuracy in space, the time evolution of the cell averages

$$
\begin{aligned}
& f_{j+1 / 2}^{r}(t)=e^{-t / \varepsilon} f_{j+1 / 2}(0)+\left(1-e^{-t / \varepsilon}\right) E_{f}\left(u_{j+1 / 2}(0)\right), \\
& g_{j+1 / 2}^{r}(t)=e^{-t / \varepsilon} g_{j+1 / 2}(0)+\left(1-e^{-t / \varepsilon}\right) E_{g}\left(u_{j+1 / 2}(0)\right) .
\end{aligned}
$$

Thus we can apply Algorithm 4.1 on the whole set of samples in the space interval interested by the reconstruction of $u_{j+1 / 2}(0)$.

For example, let us denote by the index $k$ the sample $\nu_{k}$ and its position $\chi_{k}$. If we use equations (14) then $u_{j+1 / 2}$ is given by the number of samples $N_{j}$ belonging to the cell $I_{j}$

$$
u_{j+1 / 2}=\frac{1}{N \Delta x} \sum_{x_{k} \in I_{j}} 1=\frac{N_{j}}{N \Delta x}
$$


and the Monte Carlo procedure is applied on such set of samples $\left\{\nu_{k} \mid \chi_{k} \in I_{j}\right\}$. In this case, when we extract a new equilibrium sample $\nu_{k}$ in the cell $I_{j}$ its position $\chi_{k}$ is taken as uniformly distributed in the cell.

By choosing a smoother reconstruction which uses additional cells we must take particles on the whole set of cells interested by the reconstruction (for example $I_{j-1}, I_{j}, I_{j+1}$ ) with a probability characterized by $W_{\Delta x}(x)$. Similarly we must distribute the position of new equilibrium samples.

Finally the transport step does not present any difficulty and can be applied without any need of meshes or reconstructions. In fact, from the exact expression of the solution $f^{c}(x, t)=f^{r}(x-\sqrt{a} t, t), g^{c}(x, t)=$ $g^{r}(x+\sqrt{a} t, t)$ we simply need to shift the position of the samples accordingly to the law

$$
\chi_{k}=\chi_{k}+\nu_{k} t, \quad \forall k .
$$

The method described above deserves some remarks.

\section{Remark 2.}

- One important aspect in the method is that we do not need to reconstruct the functions $f$ and $g$ but only the conserved quantity $u$. This is of paramount relevance when dealing with very large systems, as in kinetic equations.

- The terminology particle and the pair $\left(\chi_{k}, \nu_{k}\right)$ is then used to denote a sample $\nu_{k}$ and its position $\chi_{k}$. This is reminiscent of the physical nature of a particle with a certain velocity and position.

- Note that as $\varepsilon \rightarrow 0$ the method becomes a Monte Carlo algorithm for the limiting scalar conservation law. This limiting method is the analogue of a kinetic particle method for the scalar conservation law [56,60].

- The simple splitting method we have described here is first order in time. The use of the exact solution of the relaxation step is not relevant, any L-stable time discretization that maintains positivity of the solution would suffice (for example the simple fully implicit Euler method). Second order Strang splitting can be implemented similarly [20,66].

\subsection{The hybrid method (HM)}

The standard hybrid method is based on the hybrid representation (22). Thus we assume our solution of the form

$$
\begin{aligned}
& f(x, t)=(1-\beta(x, t)) f_{p}(x, t)+\beta(x, t) E_{f}(u(x, t)) \\
& g(x, t)=(1-\beta(x, t)) g_{p}(x, t)+\beta(x, t) E_{g}(u(x, t))
\end{aligned}
$$

From the exact solution of the relaxation step (24) if we consider that initially

$$
\begin{aligned}
& f(x, 0)=(1-\beta(x, 0)) f_{p}(x, 0)+\beta(x, 0) E_{f}(u(x, 0)), \\
& g(x, 0)=(1-\beta(x, 0)) g_{p}(x, 0)+\beta(x, 0) E_{g}(u(x, 0))
\end{aligned}
$$

we obtain the identities

$$
\begin{aligned}
f^{r}(x, t) & =\left(1-\beta^{r}(x, t)\right) f_{p}^{r}(x, t)+\beta^{r}(x, t) E_{f}\left(u^{r}(x, t)\right) \\
& =e^{-t / \varepsilon}\left[(1-\beta(x, 0)) f_{p}(x, 0)+\beta(x, 0) E_{f}(u(x, 0))\right]+\left(1-e^{-t / \varepsilon}\right) E_{f}(u(x, 0)) \\
g^{r}(x, t) & =\left(1-\beta^{r}(x, t)\right) g_{p}^{r}(x, t)+\beta^{r}(x, t) E_{g}\left(u^{r}(x, t)\right) \\
& =e^{-t / \varepsilon}\left[(1-\beta(x, 0)) g_{p}(x, 0)+\beta(x, 0) E_{g}(u(x, 0))\right]+\left(1-e^{-t / \varepsilon}\right) E_{g}(u(x, 0)) .
\end{aligned}
$$

By equating the equilibrium terms and the non equilibrium ones in the above equations and using the fact that $u^{r}(x, t)=u(x, 0)$ we obtain the evolution for the unknowns $f_{p}^{r}(x, t), g_{p}^{r}(x, t)$ and $\beta^{r}(x, t)$

$$
f_{p}^{r}(x, t)=f_{p}(x, 0), \quad g_{p}^{r}(x, t)=g_{p}(x, 0), \quad \beta^{r}(x, t)=e^{-t / \varepsilon} \beta(x, 0)+1-e^{-t / \varepsilon} .
$$


Note that $\beta^{r}(x, t) \rightarrow 1$ as $\varepsilon \rightarrow 0$. If we start from $\beta(x, 0)=0$ (all particles) at the end of the relaxation a fraction $1-e^{-t / \varepsilon}$ of the particles is discarded by the method as the effect of the relaxation to equilibrium. Thus particles will represent the fractions $f_{p}^{r}(x, t)=\left(1-\beta^{r}(x, t)\right) f_{p}^{r}(x, t)$ and $\left(1-\beta^{r}(x, t)\right) g_{p}^{r}(x, t)$. Moreover the hybrid representation is naturally kept by the relaxation.

After relaxation the exact solution of the transport step reads

$$
\begin{aligned}
f^{c}(x, t) & =\left(1-\beta^{c}(x, t)\right) f_{p}^{c}(x, t)+\beta^{c}(x, t) E_{f}\left(u^{c}(x, t)\right)=f^{r}(x-\sqrt{a} t, t) \\
& =\left(1-\beta^{r}(x-\sqrt{a} t, t)\right) f_{p}^{r}(x-\sqrt{a} t, t)+\beta^{r}(x-\sqrt{a} t, t) E_{f}(u(x-\sqrt{a} t, 0)) \\
g^{c}(x, t) & =\left(1-\beta^{c}(x, t)\right) g_{p}^{c}(x, t)+\beta^{c}(x, t) E_{f}\left(u^{c}(x, t)\right)=g^{r}(x+\sqrt{a} t, t) \\
& =\left(1-\beta^{r}(x+\sqrt{a} t, t)\right) g_{p}^{r}(x+\sqrt{a} t, t)+\beta^{r}(x+\sqrt{a} t, t) E_{g}(u(x+\sqrt{a} t, 0)) .
\end{aligned}
$$

To simplify notations let us set

$$
\begin{array}{ll}
f_{p}^{*}(x, t)=\left(1-\beta^{r}(x-\sqrt{a} t, t)\right) f_{p}^{r}(x-\sqrt{a} t, t), & E_{f}^{*}(x, t)=\beta^{r}(x-\sqrt{a} t, t) E_{f}(u(x-\sqrt{a} t, 0)), \\
g_{p}^{*}(x, t)=\left(1-\beta^{r}(x+\sqrt{a} t, t)\right) g_{p}^{r}(x+\sqrt{a} t, t), & E_{g}^{*}(x, t)=\beta^{r}(x+\sqrt{a} t, t) E_{g}(u(x+\sqrt{a} t, 0)) .
\end{array}
$$

Unfortunately now the hybrid structure of the solution is not kept since $E_{f}^{*}(x, t)$ and $E_{g}^{*}(x, t)$ are not equilibrium states. For example the above set of equations can be solved taking

$$
\beta^{c}(x, t)=0
$$

and

$$
\begin{aligned}
& f^{c}(x, t)=f_{p}^{*}(x, t)+E_{f}^{*}(x, t), \\
& g^{c}(x, t)=g_{p}^{*}(x, t)+E_{g}^{*}(x, t) .
\end{aligned}
$$

Thus we need to resample the whole deterministic fraction $E_{f}^{*}(x, t)$ and $E_{g}^{*}(x, t)$.

Note however that if we move one step $t_{1}$ further in the relaxation using $f^{c}(x, t)$ and $g^{c}(x, t)$ defined above as initial data we have $\beta^{r}\left(x, t+t_{1}\right)=1-e^{-t_{1} / \varepsilon}$ and

$$
\begin{aligned}
f^{r}\left(x, t+t_{1}\right) & =\left(1-\beta^{r}\left(x, t+t_{1}\right)\right) f_{p}^{r}\left(x, t+t_{1}\right)+\beta^{r}\left(x, t+t_{1}\right) E_{f}\left(u^{r}\left(x, t+t_{1}\right)\right) \\
& =e^{-t_{1} / \varepsilon} f^{c}(x, t)+\left(1-e^{-t_{1} / \varepsilon}\right) E_{f}\left(u^{c}(x, t)\right) \\
& =e^{-t_{1} / \varepsilon}\left(f_{p}^{*}(x, t)+E_{f}^{*}(x, t)\right)+\left(1-e^{-t_{1} / \varepsilon}\right) E_{f}\left(u^{c}(x, t)\right), \\
g^{r}\left(x, t+t_{1}\right) & =\left(1-\beta^{r}\left(x, t+t_{1}\right)\right) g_{p}^{r}\left(x, t+t_{1}\right)+\beta^{r}\left(x, t+t_{1}\right) E_{g}\left(u^{r}\left(x, t+t_{1}\right)\right) \\
& =e^{-t_{1} / \varepsilon} g^{c}(x, t)+\left(1-e^{-t_{1} / \varepsilon}\right) E_{g}\left(u^{c}(x, t)\right) \\
& =e^{-t_{1} / \varepsilon}\left(g_{p}^{*}(x, t)+E_{g}^{*}(x, t)\right)+\left(1-e^{-t_{1} / \varepsilon}\right) E_{g}\left(u^{c}(x, t)\right) .
\end{aligned}
$$

Thus, in practice, we can avoid to resample particles after the convection and apply the resampling only on a fraction $e^{-t_{1} / \varepsilon}$ of the deterministic fraction as needed by the relaxation. More precisely taking cell averages of (37)-(38) as in a standard Monte Carlo method, and using equations (14) for the reconstruction, the algorithm to compute the particles that represent the fractions $e^{-t_{1} / \varepsilon} f_{j+1 / 2}^{c}(t)$ and $e^{-t_{1} / \varepsilon} g_{j+1 / 2}^{c}(t)$ reads as follows

Algorithm 4.2 (Hybrid Monte Carlo for Jin-Xin relaxation).

(1) Given $m=\frac{\Delta x}{N} \sum_{j} u_{j+1 / 2}^{c}(t)$

(2) for each interval $I_{j}, j=\ldots,-2,-1,0,1,2, \ldots$ 
(a) $\operatorname{set} \beta_{j}=1-e^{-t_{1} / \varepsilon}$

(b) set $N_{j}=$ Iround $\left(\left(1-\beta_{j}\right) \frac{\Delta x}{m} u_{j+1 / 2}^{c}(t)\right)$

(c) set $P_{j}=\frac{u_{p, j+1 / 2}^{*}(t)}{u_{p, j+1 / 2}^{*}(t)+u_{E, j+1 / 2}^{*}(t)}$, with $u_{p, j+1 / 2}^{*}(t)=f_{p, j+1 / 2}^{*}(t)+g_{p, j+1 / 2}^{*}(t)$ and $u_{E, j+1 / 2}^{*}(t)=E_{f, j+1 / 2}^{*}(t)+E_{g, j+1 / 2}^{*}(t)$

(d) for $k=1, \ldots, N_{j}$ with probability $P_{j}$ take $\left(\nu_{k}, \chi_{k}\right)$ as one of the advected particles. with probability $1-P_{j}$ take one sample $\nu_{k}$ from the deterministic fraction. To extract such a sample do the following

(i) with probability $\frac{E_{f, j+1 / 2}^{*}(t)}{u_{E, j+1 / 2}^{*}}$ take $\nu_{k}=\sqrt{a}$

(ii) with probability $\frac{E_{g, j+1 / 2}^{*}(t)}{u_{E, j+1 / 2}^{*}(t)}$ take $\nu_{k}=-\sqrt{a}$

(iii) take $\chi_{k}$ uniformly distributed in $I_{j}$

After this the hybrid solution is computed simply adding the deterministic terms

$$
\beta_{j} E_{f}\left(u_{j+1 / 2}^{c}(t)\right), \quad \beta_{j} E_{g}\left(u_{j+1 / 2}^{c}(t)\right)
$$

to the stochastic terms

$$
\left(1-\beta_{j}\right) f_{p, j+1 / 2}^{r}(t)=\frac{m}{\Delta x} N_{j}^{+}, \quad\left(1-\beta_{j}\right) g_{p, j+1 / 2}^{r}(t)=\frac{m}{\Delta x} N_{j}^{-}
$$

where $N_{j}^{+}$and $N_{j}^{-}$are the number of samples in cell $I_{j}$ equal to $\sqrt{a}$ and $-\sqrt{a}$ respectively.

This permits to avoid inefficient discard-resample procedures for small values of $\varepsilon$. For example, as $\varepsilon \rightarrow 0$ we do not perform any resampling at all, and we obtain a relaxation scheme for the limiting scalar conservation law.

\section{Remark 3.}

- The convection part corresponding to $f_{p}^{*}(x, t)$ and $g_{p}^{*}(x, t)$ is solved exactly by transport of particles as in a full Monte Carlo method. At variance the convection part corresponding to $E_{f}^{*}(x, t)$ and $E_{g}^{*}(x, t)$ can be solved by finite volumes or finite differences since it corresponds to solve the convection step with initial data $f(x, 0)=\beta^{r}(x, t) E_{f}(u(x, 0))$ and $g(x, 0)=\beta^{r}(x, t) E_{g}(u(x, 0))$. In practice this is equivalent to solve a relaxation scheme for the limiting conservation law.

- Note that the effective value of $\beta_{j}$ used in the above algorithm differs from $1-e^{-t_{1} / \varepsilon}$. In fact if $N_{j}^{c}$ denotes the number of particles in cell $j$ after the convection step, during the relaxation we keep only an integer approximation $N_{j}^{\beta}$ of $\left(1-\beta_{j}\right) N_{j}^{c}$. The effective value of $\beta_{j}$ can then be computed at the end of the algorithm as

$$
\beta_{j}^{E}=1-\frac{N_{j}^{\beta}}{N_{j}^{c}} .
$$

\subsection{A Componentwise hybrid method (CHM)}

A better approach would consist in finding the maximum value of $\beta^{c}(x, t)>0$ in order to maximize the deterministic fraction in equations (33). In order to achieve this goal we consider the componentwise hybrid representation

$$
\begin{aligned}
f(x, t) & =\tilde{f}(x, t)+w_{f}(x, t) E_{f}(u(x, t)) \\
g(x, t) & =\tilde{g}(x, t)+w_{g}(x, t) E_{g}(u(x, t)) .
\end{aligned}
$$


The relaxation step now leads to

$$
\begin{aligned}
f^{r}(x, t) & =\tilde{f}^{r}(x, t)+w_{f}^{r}(x, t) E_{f}\left(u^{r}(x, t)\right) \\
& =e^{-t / \varepsilon}\left[\tilde{f}(x, 0)+w_{f}(x, 0) E_{f}(u(x, 0))\right]+\left(1-e^{-t / \varepsilon}\right) E_{f}(u(x, 0)) \\
g^{r}(x, t) & =\tilde{g}^{r}(x, t)+w_{g}^{r}(x, t) E_{g}\left(u^{r}(x, t)\right) \\
& =e^{-t / \varepsilon}\left[\tilde{g}(x, 0)+w_{g}(x, 0) E_{g}(u(x, 0))\right]+\left(1-e^{-t / \varepsilon}\right) E_{g}(u(x, 0)) .
\end{aligned}
$$

Again by equating the equilibrium terms and the non equilibrium ones in the above equations we obtain the evolution for the unknowns $\tilde{f}^{r}(x, t), \tilde{g}^{r}(x, t), w_{f}^{r}(x, t)$ and $w_{g}^{r}(x, t)$

$$
\begin{array}{ll}
\tilde{f}^{r}(x, t)=e^{-t / \varepsilon} \tilde{f}(x, 0), & w_{f}^{r}(x, t)=e^{-t / \varepsilon} w_{f}(x, 0)+1-e^{-t / \varepsilon} \\
\tilde{g}^{r}(x, t)=e^{-t / \varepsilon} \tilde{g}(x, 0), & w_{g}^{r}(x, t)=e^{-t / \varepsilon} w_{g}(x, 0)+1-e^{-t / \varepsilon}
\end{array}
$$

As before the hybrid representation is kept by the relaxation process. The only difference with respect to the HM method is that we discard particles from $f$ and $g$ with different ratios.

The convection destroys the structure of the solution and we get

$$
\begin{aligned}
f^{c}(x, t) & =\tilde{f}^{c}(x, t)+w_{f}^{c}(x, t) E_{f}\left(u^{c}(x, t)\right)=f^{r}(x-\sqrt{a} t, t) \\
& =\tilde{f}^{r}(x-\sqrt{a} t, t)+w_{f}^{r}(x-\sqrt{a} t, t) E_{f}(u(x-\sqrt{a} t, 0)) \\
g^{c}(x, t) & =\tilde{g}^{c}(x, t)+w_{g}^{c}(x, t) E_{f}\left(u^{c}(x, t)\right)=g^{r}(x+\sqrt{a} t, t) \\
& =\tilde{g}^{r}(x+\sqrt{a} t, t)+w_{g}^{r}(x+\sqrt{a} t, t) E_{g}(u(x+\sqrt{a} t, 0)) .
\end{aligned}
$$

To simplify notations let us set

$$
\begin{array}{ll}
f_{p}^{*}(x, t)=\tilde{f}^{r}(x-\sqrt{a} t, t), & \tilde{E}_{f}(x, t)=w_{f}^{r}(x-\sqrt{a} t, t) E_{f}(u(x-\sqrt{a} t, 0)), \\
g_{p}^{*}(x, t)=\tilde{g}^{r}(x+\sqrt{a} t, t), & \tilde{E}_{g}(x, t)=w_{g}^{r}(x+\sqrt{a} t, t) E_{g}(u(x+\sqrt{a} t, 0)) .
\end{array}
$$

Here we do not assume $w_{f}^{c}(x, t)=0, w_{g}^{c}(x, t)=0$ since we want to take advantage of the componentwise hybrid representation in order to maximize the deterministic fraction of the solution. Thus, starting from the deterministic fractions $\tilde{E}_{f}(x, t)$ and $\tilde{E}_{g}(x, t)$ defined above we construct the new values of $w_{f}^{c}(x, t), \tilde{f}^{c}(x, t)$, $w_{g}^{c}(x, t)$ and $\tilde{g}^{c}(x, t)$ using Definition 1.

More precisely we define

$$
w_{f}^{c}(x, t)= \begin{cases}\frac{\tilde{E}_{f}(x, t)}{E_{f}\left(u^{c}(x, t)\right)}, & \tilde{E}_{f}(x, t) \leq E_{f}\left(u^{c}(x, t)\right) \neq 0 \\ 1, & \tilde{E}_{f}(x, t) \geq E_{f}\left(u^{c}(x, t)\right)\end{cases}
$$

and

$$
E_{f}^{*}(x, t)=\tilde{E}_{f}(x, t)-w_{f}^{c}(x, t) E_{f}\left(u^{c}(x, t)\right)
$$

In this way we obtain

$$
\tilde{f}^{c}(x, t)=f_{p}^{*}(x, t)+E_{f}^{*}(x, t) .
$$

Note that if $\tilde{E}_{f}(x, t) \leq E_{f}\left(u^{c}(x, t)\right) \neq 0$ we have $\tilde{f}^{c}(x, t)=f_{p}^{*}(x, t)$ and thus we keep all the deterministic fraction. Similarly we compute $w_{g}^{c}(x, t)$ and $\tilde{g}_{p}^{c}(x, t)$. 
The next relaxation step then applies straightforwardly using directly Algorithm 4.2 on cell averages. In fact moving one step further we have

$$
\begin{aligned}
f^{r}\left(x, t+t_{1}\right) & =\tilde{f}^{r}\left(x, t+t_{1}\right)+w_{f}^{r}\left(x, t+t_{1}\right) E_{f}\left(u^{r}\left(x, t+t_{1}\right)\right) \\
& =e^{-t_{1} / \varepsilon} f^{c}(x, t)+\left(1-e^{-t_{1} / \varepsilon}\right) E_{f}\left(u^{c}(x, t)\right) \\
& =e^{-t_{1} / \varepsilon}\left(f_{p}^{*}(x, t)+E_{f}^{*}(x, t)+w_{f}(x, t) E_{f}(x, t)\right)+\left(1-e^{-t_{1} / \varepsilon}\right) E_{f}\left(u^{c}(x, t)\right) \\
g^{r}\left(x, t+t_{1}\right) & =\tilde{g}^{r}\left(x, t+t_{1}\right)+w_{g}^{r} E_{g}\left(u^{r}\left(x, t+t_{1}\right)\right) \\
& =e^{-t_{1} / \varepsilon} g^{c}(x, t)+\left(1-e^{-t_{1} / \varepsilon}\right) E_{g}\left(u^{c}(x, t)\right) \\
& =e^{-t_{1} / \varepsilon}\left(g_{p}^{*}(x, t)+E_{g}^{*}(x, t)+w_{g}(x, t) E_{g}(x, t)\right)+\left(1-e^{-t_{1} / \varepsilon}\right) E_{g}\left(u^{c}(x, t)\right) .
\end{aligned}
$$

The only difference is that now the final hybrid solution is recovered adding the deterministic terms

$$
\left(\left(1-\beta_{j}\right) w_{f}\left(x_{j+1 / 2}, t\right)+\beta_{j}\right) E_{f}\left(u_{j+1 / 2}^{c}(t)\right), \quad\left(\left(1-\beta_{j}\right) w_{g}\left(x_{j+1 / 2}, t\right)+\beta_{j}\right) E_{g}\left(u_{j+1 / 2}^{c}(t)\right)
$$

in each cell.

\section{Remark 4.}

- If we define after the convection step

$$
\beta^{c}(x, t)=\min \left\{w_{f}^{c}(x, t), w_{g}^{c}(x, t)\right\},
$$

we maximize the common value of $\beta^{c}$ such that the standard hybrid method applies. This is particularly relevant in many applications where it is important that the hybrid decomposition is component independent. For example for more general relaxation terms.

- More in general a hybrid method can be formulated as follows. Starting from a general nonequilibrium/equilibrium decomposition of the solution in the form $f=\tilde{f}+f_{E}$ and $g=\tilde{g}+g_{E}$ we solve separately in each time step

$$
\begin{aligned}
\partial_{t} \tilde{f}+\sqrt{a} \partial_{x} \tilde{f} & =-\frac{1}{\varepsilon} \tilde{f} \\
\partial_{t} \tilde{g}-\sqrt{a} \partial_{x} \tilde{g} & =-\frac{1}{\varepsilon} \tilde{g},
\end{aligned}
$$

using a Monte Carlo strategy and

$$
\begin{aligned}
\partial_{t} f_{E}+\sqrt{a} \partial_{x} f_{E} & =-\frac{1}{\varepsilon}\left(f_{E}-E_{f}(u)\right) \\
\partial_{t} g_{E}-\sqrt{a} \partial_{x} g_{E} & =-\frac{1}{\varepsilon}\left(g_{E}-E_{g}(u)\right),
\end{aligned}
$$

with a deterministic scheme. At the end the solution is projected back to the form $f=\tilde{f}+f_{E}$ and $g=\tilde{g}+g_{E}$ using a suitable representation step. Note that taking initially $f_{E}=E_{f}(u)$ and $g_{E}=E_{g}(u)$, by allowing $\tilde{f}$ and $\tilde{g}$ to assume negative values, corresponds exactly to solve a relaxed scheme for the limiting conservation law.

\section{IMPLEMENTATION AND NUMERICAL TESTS}

In this section we report some numerical results for the different schemes considered. We use the shorthand MCM, HM1, HM2 and CHM to denote the Monte Carlo Method, the Hybrid Method with the choices (34) and (50) respectively, and the Componentwise Hybrid Method. 


\subsection{Solving the equilibrium part}

In order to determine the evolution of the deterministic part of the solution in the hybrid methods we use a second order MUSCL type scheme based on cell averages [41]. The second order scheme is defined taking as a flux

$$
F(f ; j)=\sqrt{a} f_{j}+\frac{1}{2} \sqrt{a}(1-\nu)\left(f_{j+1}-f_{j}\right) \phi_{j},
$$

where $\nu=\sqrt{a} \Delta t / \Delta x$ and $\phi_{j}$ is the limiter function

$$
\phi_{j}=\phi\left(\theta_{j}\right), \quad \theta_{j}=\frac{f_{j}-f_{j-1}}{f_{j+1}-f_{j}} .
$$

For example the so-called "superbee" of Roe

$$
\phi(\theta)=\max (0, \min (1,2 \theta), \min (\theta, 2)) .
$$

Finally the scheme for the convection step is defined as

$$
\begin{aligned}
f_{j}^{n+1} & =f_{j}^{n}-\nu\left(f_{j}^{n}-f_{j-1}^{n}\right)-\frac{1}{2} \nu(1-\nu)\left(f_{j+1}^{n}-f_{j}^{n}\right) \phi\left(\theta_{j}\right) \\
& +\frac{1}{2} \nu(1-\nu)\left(f_{j}^{n}-f_{j-1}^{n}\right) \phi\left(\theta_{j-1}\right) \\
g_{j}^{n+1} & =g_{j}^{n}-\nu\left(g_{j}^{n}-g_{j-1}^{n}\right)-\frac{1}{2} \nu(1-\nu)\left(g_{j+1}^{n}-g_{j}^{n}\right) \phi\left(\theta_{j}\right) \\
& +\frac{1}{2} \nu(1-\nu)\left(g_{j}^{n}-g_{j-1}^{n}\right) \phi\left(\theta_{j-1}\right)
\end{aligned}
$$

As $\varepsilon \rightarrow 0$ the relaxation step becomes a projection step and thus we obtain a second order in space, first order in time relaxation scheme [36] for the limiting scalar conservation law. Extension to the multidimensional case can be done as usual dimension by dimension.

\subsection{Numerical examples}

In all tests we take initially the solution represented by samples and $F(u)=u^{2} / 2$ (thus as $\varepsilon \rightarrow 0$ we have the Burgers equation). We consider the following test cases with periodic boundary conditions.

\subsection{1. $1 D$ case}

First we consider the one-dimensional problem

$$
u(x, 0)=\frac{1}{4}(2+\sin (2 \pi x)-\sin (\pi x)), \quad x \in[-1,1], \quad t \in[0,1]
$$

We report the numerical solution for different values of the relaxation parameter $\varepsilon=0.1,0.01,0.001$ starting initially with $N=10^{3}$ particles. We use 50 grid points for $\varepsilon=0.1$ and $\varepsilon=0.01$ and 100 grid points for $\varepsilon=0.001$. The particle solution has been reconstructed using the simple formula (14). The final computing times are given in the figures captions (see Figures 2 and 3). For each test case we also compute the $L_{1}$ norm of the error in time using a finite difference solution on a very fine mesh as a reference result (see Figure 4).

In the same figure the number of particles as a function of time is also given. The variance reduction of hybrid methods with respect to standard MCM is evident. In particular HM2 and CHM have the better efficiency and accuracy properties. 

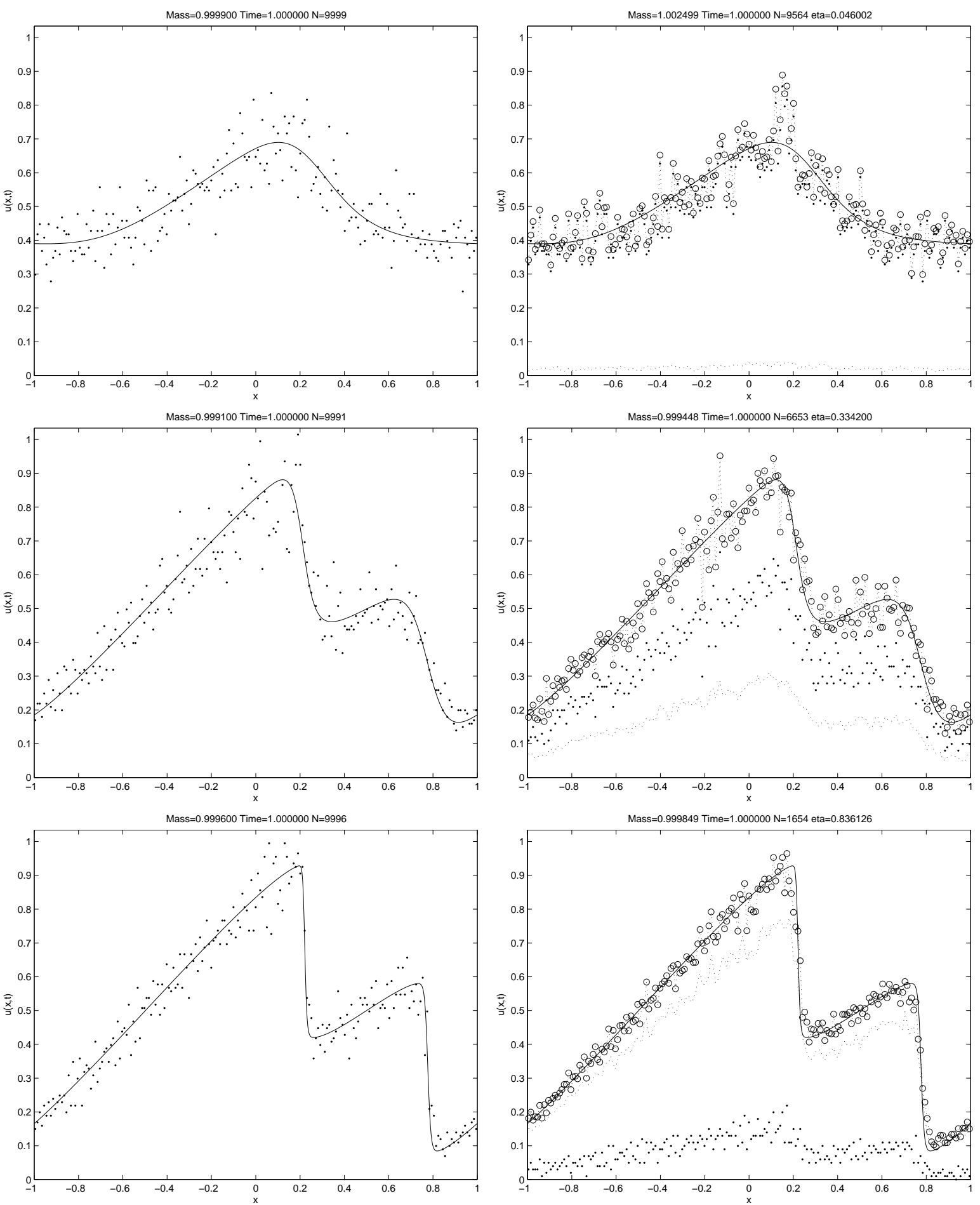

Figure 2. $1 \mathrm{D}$ case: Solution at $t=1$ with $\varepsilon=0.1$ (top), $\varepsilon=0.01$ (middle) and $\varepsilon=0.001$ (bottom) for MCM (left) and HM1 (right). Particle solution (·), equilibrium solution (dashed line) and hybrid solution (o). 

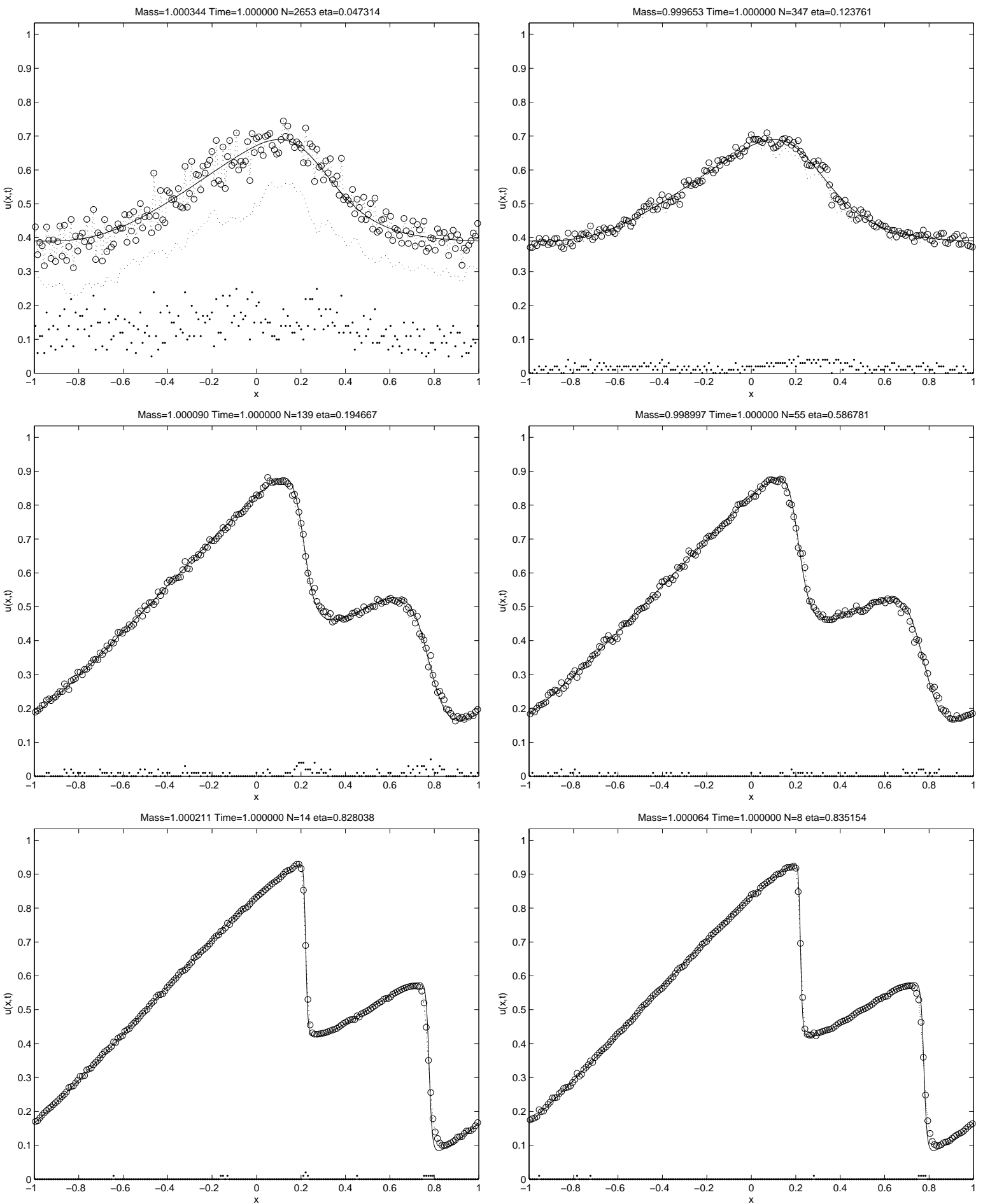

Figure 3. 1D case: Solution at $t=1$ with $\varepsilon=0.1$ (top), $\varepsilon=0.01$ (middle) and $\varepsilon=0.001$ (bottom) for HM2 (left) and CHM (right). Particle solution (·), equilibrium solution (dashed line) and hybrid solution (o). 

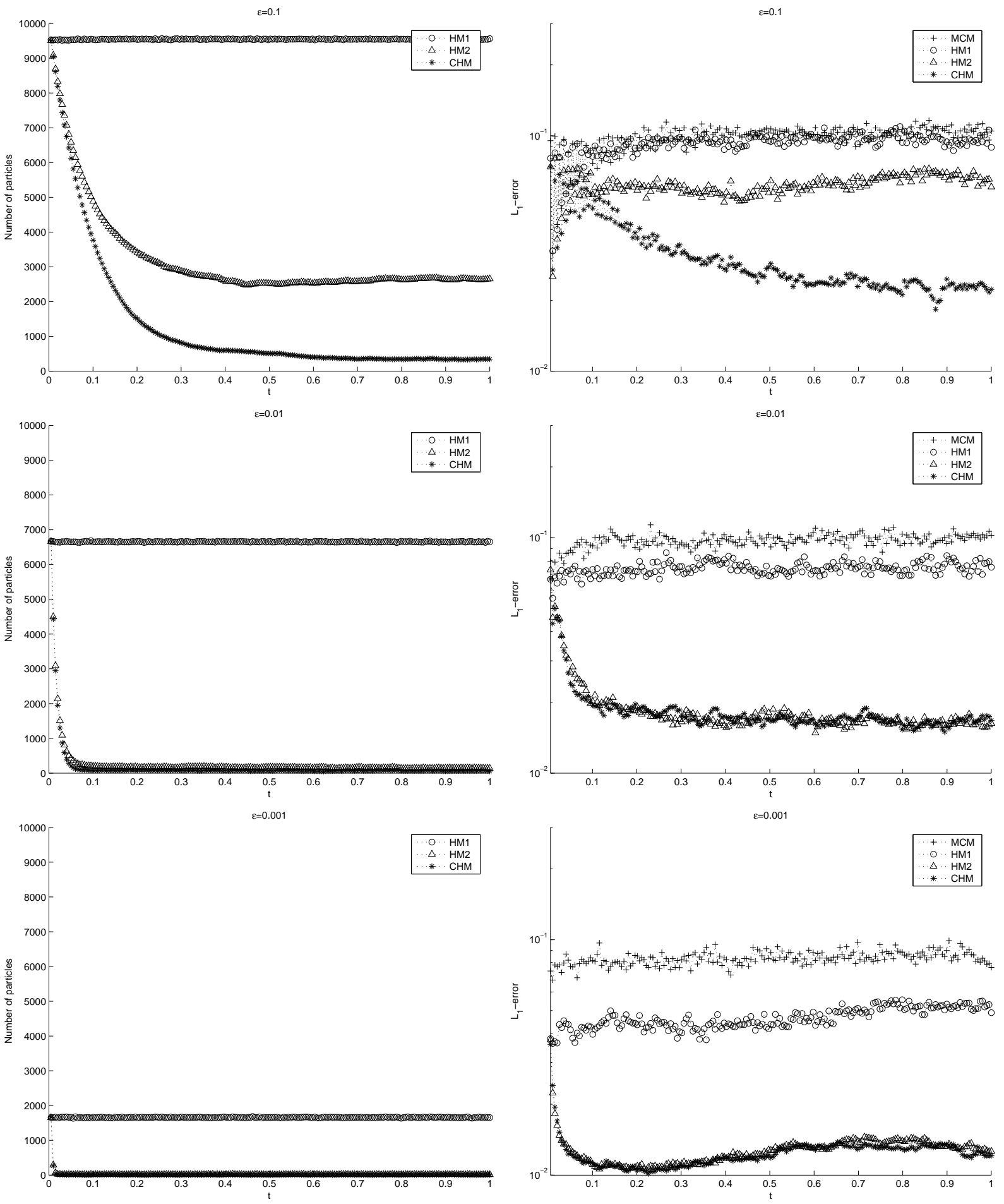

Figure 4. 1D case: Number of particles (left) and relative $L_{1}$-error in time (right) for $\varepsilon=0.1$ (top), $\varepsilon=0.01$ (middle) and $\varepsilon=0.001$ (bottom). 


\subsubsection{D case}

Next we consider the $2 \mathrm{D}$ case with periodic boundary condition and initial data

$$
u(x, y)=\sin (\pi x)^{2} \sin (\pi y)^{2}, \quad(x, y) \in[0,1]^{2} .
$$

We report the result only for $H M 2$ method and $\varepsilon=0.1$ and $\varepsilon=0.01$ using a $40 \times 40$ mesh. The initial data is represented by $N=4 \times 10^{4}$ particles. The results and the final computation times are shown in Figure 5. Finally we also report the result obtained for $\varepsilon=10^{-6}$ with a $80 \times 80$ mesh. Due to the small value of $\varepsilon$ all hybrid methods yield essentially the same result corresponding to a second order relaxation scheme for the limiting equation.

\section{Kinetic EQUATions}

The methods developed in the previous sections can be extended directly to kinetic equations of the form $[13,14]$

$$
\partial_{t} f+v \nabla_{x} f=\frac{1}{\varepsilon} Q(f, f)
$$

Here $f=f(x, v, t) \geq 0$ is the gas particle density, $x, v \in \mathbb{R}^{d}, d \geq 1$, and the collision term $Q(f, f)$ describes the interactions between particles. In the case of the Boltzmann operator of rarefied gas dynamics it is well known that any equilibrium distribution function, i.e. any function $f$ for which $Q(f, f)=0$, has the form of a locally Maxwellian distribution

$$
M(\rho, u, T)(v)=\frac{\rho}{(2 \pi T)^{d / 2}} \exp \left(-\frac{|u-v|^{2}}{2 T}\right),
$$

where $\rho, u, T$ are the mass density, mean velocity and temperature of the gas defined by

$$
\rho=\int_{\mathbb{R}^{d}} f d v, \quad u=\frac{1}{\rho} \int_{\mathbb{R}^{d}} v f d v, \quad T=\frac{1}{d \rho} \int_{\mathbb{R}^{d}}[v-u]^{2} f d v .
$$

As $\varepsilon \rightarrow 0$ the distribution function approaches the local Maxwellian (51). In this case the higher order moments of the distribution $f$ can be computed as function of $\rho, u$, and $T$, by using (51) and we obtain to the leading order the closed system of compressible Euler equations of gas dynamics

$$
\begin{aligned}
& \frac{\partial \rho}{\partial t}+\nabla_{x} \cdot(\rho u)=0 \\
& \frac{\partial \rho u}{\partial t}+\nabla_{x} \cdot(\rho u \otimes u+p)=0 \\
& \frac{\partial E}{\partial t}+\nabla_{x} \cdot(E u+p u)=0 \\
& p=\rho T, \quad E=\frac{d}{2} \rho T+\frac{1}{2} \rho u^{2} .
\end{aligned}
$$

where $p$ is the gas pressure.

Here we discuss only the development of hybrid methods we refer to [55] and the references therein for an introduction to Monte Carlo methods for kinetic equations.

In order to do that we introduce the following

Definition 2. Given a probability density $f(v), v \in \mathbb{R}^{d}$ (i.e. $f(v) \geq 0, \int f(v) d v=1$ ) and a probability density $M(v), v \in \mathbb{R}^{d}$ called equilibrium density, we define $w(v) \in[0,1]$ and $\tilde{f}(v) \geq 0$ in the following way

$$
w(v)= \begin{cases}\frac{f(v)}{M(v)}, & f(v) \leq M(v) \neq 0 \\ 1, & f(v) \geq M(v)\end{cases}
$$



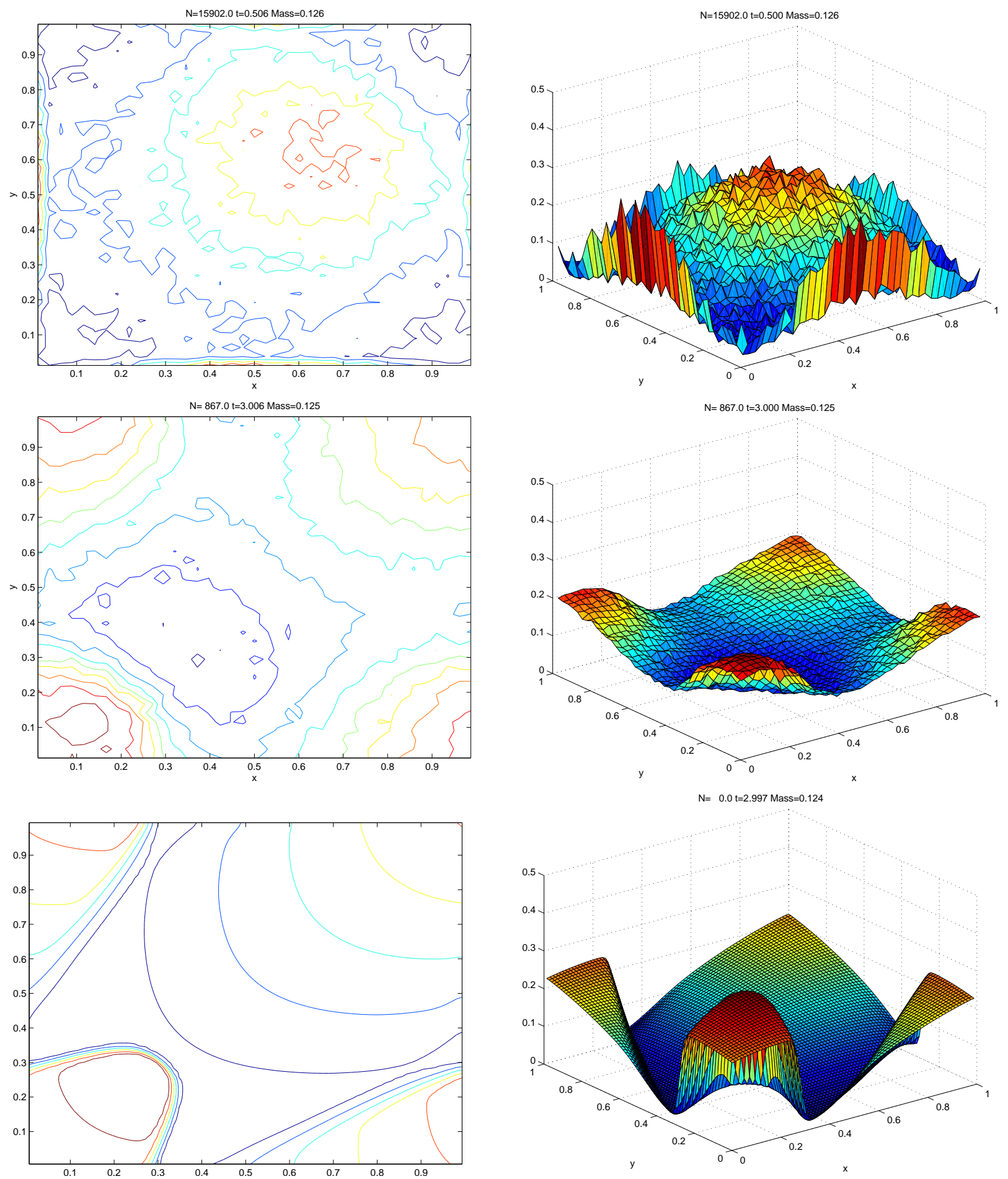

Figure 5. 2D case: Solution at $t=0.5$ with $\varepsilon=0.1$ (top) and at $t=3.0$ with $\varepsilon=0.01$ (middle) and $\varepsilon=0.001$ (bottom) for HM. 

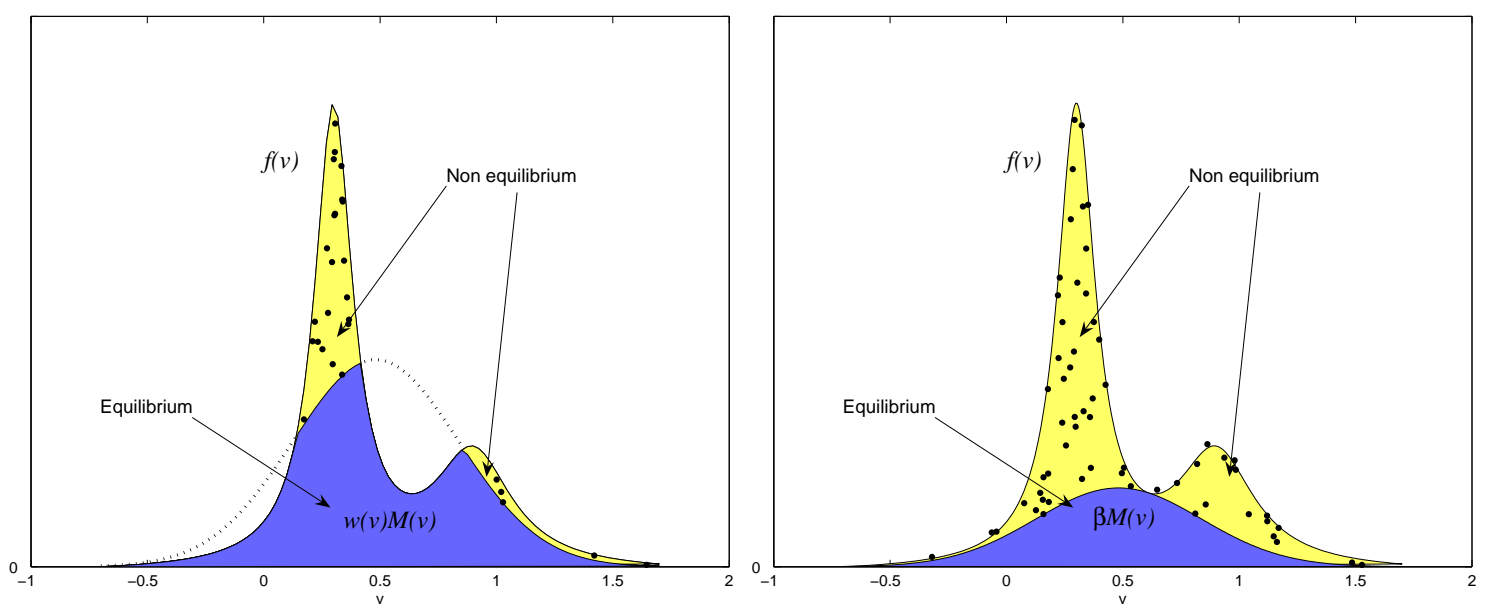

FiguRE 6. Hybrid representation of a probability density in the form (57) left and (61) right.

and

Thus $f(v)$ can be represented as

$$
\tilde{f}(v)=f(v)-w(v) M(v) .
$$

$$
f(v)=\tilde{f}(v)+w(v) M(v) .
$$

Remark 5. Taking

$$
\beta=\min _{v}\{w(v)\}
$$

and

we have

$$
\tilde{f}(v)=f(v)-\beta M(v),
$$

$$
\int \tilde{f}(v) d v=1-\beta .
$$

Let us define for $\beta \neq 1$ the probability density

$$
f_{p}(v)=\frac{\tilde{f}(v)}{1-\beta}
$$

The case $\beta=1$ is trivial since it implies $f \equiv M$. Thus we recover the hybrid representation introduced in [51,52] as

$$
f(v)=(1-\beta) f_{p}(v)+\beta M(v) .
$$

\subsection{BGK models}

For BGK-like kinetic equations the collision term has the form of a relaxation operator

$$
Q(f, f)=(M(f)-f)
$$

where $M(f)$ is the local Maxwellian equilibrium (51) associated with mass density, mean velocity and temperature. The extension of the hybrid methods to such kinetic models is rather straightforward and does not present particular difficulties. Again the methods are based on a time splitting of the equation and on the 
hybrid representation of standard type (61) or the velocity dependent one (57). The main differences are the following

- the sample values $\nu_{k}$ now can take any value in $\mathbb{R}^{d}$,

- the treatment of the continuum part requires a suitable approximation grid (and typically the introduction of artificial boundaries) in the velocity space.

We give details for the general velocity dependent hybrid representation (57) which can be easily reduced to the standard case (61). Thus we start by assuming

$$
f(x, v, t)=\tilde{f}(x, v, t)+w(x, v, t) M(f)(x, v, t) .
$$

The relaxation step

$$
\partial_{t} f^{r}(x, v, t)=\frac{1}{\varepsilon}\left(M\left(f^{r}\right)(x, v, t)-f^{r}(x, v, t)\right),
$$

starting from (62) at $t=0$ can be solved exactly thanks to the conservation of mass density, momentum and energy and writes as

$$
\begin{aligned}
f^{r}(x, v, t) & =\tilde{f}^{r}(x, v, t)+w^{r}(x, v, t) M\left(f^{r}\right)(x, v, t) \\
& =e^{-t / \varepsilon}[\tilde{f}(x, v, 0)+w(x, v, 0) M(f)(x, v, 0)]+\left(1-e^{-t / \varepsilon}\right) M(f)(x, v, 0) .
\end{aligned}
$$

As usual, since $M\left(f^{r}\right)(x, v, t)=M(f)(x, v, 0)$, by equating the equilibrium terms and the non equilibrium ones in the above equations we obtain

$$
\tilde{f}^{r}(x, v, t)=e^{-t / \varepsilon} \tilde{f}(x, v, 0), \quad w^{r}(x, v, t)=e^{-t / \varepsilon} w(x, v, 0)+1-e^{-t / \varepsilon} .
$$

Thus the hybrid representation is still kept at the next time level. At variance convection

$$
\partial_{t} f^{c}(x, v, t)+v \nabla_{x} f^{c}(x, v, t)=0,
$$

destroys the solution structure and gives at the next step

$$
\begin{aligned}
f^{c}(x, v, t) & =\tilde{f}^{r}(x-v t, v, t)+w^{r}(x-v t, v, t) M(f)(x-v t, v, 0) \\
& =e^{-t / \varepsilon}[\tilde{f}(x-v t, v, 0)+w(x-v t, v, 0) M(f)(x-v t, v, 0)] \\
& +\left(1-e^{-t / \varepsilon}\right) M(f)(x-v t, v, 0)
\end{aligned}
$$

To simplify notations let us set

$$
\left.f_{p}^{*}(x, v, t)=\tilde{f}^{r}(x-v t, v, t), \quad \tilde{M}(x, v, t)=w^{r}(x-v t, v, t) M(f)(x-v t, v, 0)\right) .
$$

Using Definition 2 on the deterministic fraction $\tilde{M}(x, v, t)$ defined above we construct the new values of $w^{c}(x, v, t), \tilde{f}^{c}(x, v, t)$.

More precisely we define

$$
w^{c}(x, v, t)= \begin{cases}\frac{\tilde{M}(x, v, t)}{M\left(f^{c}\right)(x, v, t)}, & \tilde{M}(x, v, t) \leq M\left(f^{c}\right)(x, v, t) \neq 0 \\ 1, & \tilde{M}(x, v, t) \geq M\left(f^{c}\right)(x, v, t)\end{cases}
$$

and

In this way we obtain

$$
M^{*}(x, v, t)=\tilde{M}(x, v, t)-w^{c}(x, v, t) M\left(f^{c}\right)(x, v, t)
$$

$$
\tilde{f}^{c}(x, v, t)=f_{p}^{*}(x, v, t)+M^{*}(x, v, t) .
$$


Thus the main structure of the method remains unchanged when compared to the case of hyperbolic relaxation system. Moving one time step $t_{1}$ further and after the introduction of cell averages in space, here we consider the one-dimensional case for simplicity, the algorithm for the relaxation reads as follows

Algorithm 6.1 (Hybrid Monte Carlo for BGK relaxation).

(1) Given $\rho_{j+1 / 2}(t)=\int_{\mathbb{R}^{d}} f_{j+1 / 2}(v, t) d v$ and $m=\frac{\Delta x}{N} \sum_{j} \rho_{j+1 / 2}(t)$

(2) for each interval $I_{j}, j=\ldots,-2,-1,0,1,2, \ldots$

(a) $\operatorname{set} \beta_{j}=1-e^{-t_{1} / \varepsilon}$

(b) set $N_{j}=$ Iround $\left(\left(1-\beta_{j}\right) \frac{\Delta x}{m} \rho_{j+1 / 2}^{c}(t)\right)$, where $\rho_{j+1 / 2}^{c}(t)=\int_{\mathbb{R}^{d}} f_{j+1 / 2}^{c}(v, t) d v$

(c) $\operatorname{set} P_{j}=\frac{\rho_{p, j+1 / 2}^{*}(t)}{\rho_{p, j+1 / 2}^{*}(t)+\rho_{M, j+1 / 2}^{*}(t)}$ with $\rho_{p, j+1 / 2}^{*}(t)=\int_{\mathbb{R}^{d}} f_{p, j+1 / 2}^{*}(v, t) d v$ and $\rho_{M, j+1 / 2}^{*}(t)=\int_{\mathbb{R}^{d}} M_{j+1 / 2}^{*}(v, t) d v$.

(d) for $k=1, \ldots, N_{j}$ with probability $P_{j}$ take $\left(\chi_{k}, \nu_{k}\right)$ as one of the advected particles in $f_{p, j+1 / 2}^{*}(v, t)$. with probability $1-P_{j}$

(i) take one sample $\nu_{k}$ from the deterministic fraction $M_{j+1 / 2}^{*}(v, t)$

(ii) take $\chi_{k}$ uniformly distributed in $I_{j}$

The above algorithm requires two further steps. First, a way to compute the mass densities in each cell defined through velocity integrals and second to specify how to sample from the deterministic fractions $M_{j+1 / 2}^{*}(v, t)$. Both aspects are strictly related to the choice of the velocity approximation method used to solve the equilibrium part.

\subsubsection{Kinetic schemes}

Following the approach of kinetic schemes one may want to preserve as much of the analytic representation as possible. The method is then based on computing the flux to cell $j$ from a Maxwellian in cell $j^{\prime}$. Here we summarize the main features of such schemes. We refer to $[19,46,59,72]$ for more details. We assume for notation clarity $x, v \in \mathbb{R}$.

Consider the cells $I_{j}=\left[x_{j}, x_{j+1}\right]$ in each of which there is a Maxwellian $M_{j+1 / 2}(v, t)$. In a time interval $[0, t]$, the range of velocities (in the x-direction) going from cell $j^{\prime}$ to cell $j$ is

$$
\left[v_{0}, v_{1}\right]=\left[\frac{\left(x_{j^{\prime}}-x_{j+1}\right)}{t}, \frac{\left(x_{j^{\prime}+1}-x_{j}\right)}{t}\right] .
$$

The resulting velocity distribution in cell $j$ that came from cell $j^{\prime}$, is the "Maxwellian hat function" $M_{j j^{\prime}}$, defined as $[10]$

$$
M_{j j^{\prime}}=h(v) M_{j^{\prime}+1 / 2}(v), \quad h(v)=\min \left(v-v_{0}, v_{1}-v\right) .
$$

At this point, cell $\mathrm{j}$ contains a collection of Maxwellian hat functions $M_{j j^{\prime}}$. Then the macroscopic density, velocity and temperature for that cell can be computed analytically by direct integration of the Maxwellian hat functions. Similarly by inverse transform methods we can sample the Maxwellian hat functions when needed.

A more general method can be constructed using a uniform mesh $v_{i}, i=\ldots,-2,-1,0,1,2, \ldots$ Let us denote with $v_{i+1 / 2}$ the center of the cell $V_{i}=\left[v_{i}, v_{i+1}\right]$ the velocity integrals that appear in Algorithm 6.1 can be recovered by quadrature, for example by the simple midpoint rule. To compute the values $M_{j+1 / 2}^{*}\left(v_{i+1 / 2}, t\right)$ one can adopt any numerical method for transport problems $[25,41]$ to get

$$
\left.\tilde{M}_{j+1 / 2}\left(v_{i+1 / 2}, t\right) \approx w^{r}\left(x_{j+1 / 2}-v_{i+1 / 2} t, v_{i+1 / 2}, t\right) M(f)\left(x_{j+1 / 2}-v_{i+1 / 2} t, v_{i+1 / 2}, 0\right)\right) .
$$


Finally to sample from the deterministic fraction once we have computed the values $M_{j+1 / 2}^{*}\left(v_{i+1 / 2}, t\right), \forall i, j$ we can take a sample $\nu_{k}$ uniformly distributed in $V_{i}$ with probability

$$
\frac{\Delta v M_{j+1 / 2}^{*}\left(v_{i+1 / 2}, t\right)}{\rho_{M, j+1 / 2}^{*}(t)}, \quad \rho_{M, j+1 / 2}^{*}(t)=\Delta v \sum_{i} M_{j+1 / 2}^{*}\left(v_{i+1 / 2}, t\right) .
$$

In both methods to compute the integrals of $f_{p, j+1 / 2}^{*}(v, t)$ in agreement with (14) we can simply sum up all particles in cell $I_{j}$.

\section{Remark 6.}

- For numerical purposes typically it is necessary to introduce artificial boundaries in the velocity space when we compute the above mentioned kinetic schemes. To avoid truncation errors for large velocities the hybrid method can be designed in such a way that the large velocity tails are always represented by particles. This corresponds to set

$$
w(x, v, t)=0, \quad|v|>R, R>0,
$$

in the definition of the hybrid solution. Note that in this case an additional representation step is required after the relaxation step since property (67) is not maintained from equations (63). We omit the technical details [21].

- A general hybrid decomposition is given by

$$
f(v)=\tilde{f}(v)+f_{E}(v)
$$

where now $\tilde{f}$ can take also negative values. Without splitting this corresponds to solve in two separate steps

by particles and

$$
\partial_{t} \tilde{f}+v \nabla_{x} \tilde{f}=-\frac{1}{\varepsilon} \tilde{f}
$$

$$
\partial_{t} f_{E}+v \nabla_{x} f_{E}=-\frac{1}{\varepsilon}\left(f_{E}-M\right)
$$

with a deterministic solver. After each time step the solution need to be projected back to the form $f(v)=$ $\tilde{f}(v)+f_{E}(v)$ using a suitable representation step. In this way taking $f_{E}(v)=M(v)$ the deterministic step is exactly a kinetic scheme for the Euler equations.

\subsection{The Boltzmann equation}

For more general collision operators, after the time splitting, the difficulty relies in the approximation of the relaxation step

$$
\partial_{t} f^{r}(x, v, t)=\frac{1}{\varepsilon} Q\left(f^{r}, f^{r}\right)(x, v, t)
$$

For small $\varepsilon$ we have a stiff source which may originate a dense nonlinear system if solved by implicit schemes. For Boltzmann like equations we have

$$
Q(f, f)(v)=\int_{\mathbb{R}^{3}} \int_{S^{2}} B\left(\left|v-v_{*}\right|, \omega\right)\left[f\left(v^{\prime}\right) f\left(v_{*}^{\prime}\right)-f(v) f\left(v_{*}\right)\right] d \omega d v_{*},
$$

where the dependence on $(x, t)$ has been omitted for notation brevity. In the above expression, $\omega$ is a unit vector of the sphere $S^{2}$, so that $d \omega$ is an element of area of the surface of the unit sphere $S^{2}$ in $\mathbb{R}^{3}$ and $B$ is 
a nonnegative kernel which characterizes the details of the binary interactions. Moreover $\left(v^{\prime}, v_{*}^{\prime}\right)$ represent the post-collisional velocities associated with the pre-collisional velocities $\left(v, v_{*}\right)$ and the collision parameter $\omega$

$$
v^{\prime}=\frac{1}{2}\left(v+v_{*}+\left|v-v_{*}\right| \omega\right), \quad v_{*}^{\prime}=\frac{1}{2}\left(v+v_{*}-\left|v-v_{*}\right| \omega\right)
$$

For such integral operator a fully implicit scheme in the collision step will originate a prohibitive computational cost. Thus the hybrid methods cannot be extend straightforwardly to the full Boltzmann equation of rarefied gas dynamics. To this goal we adopted the Time Relaxed (TR) schemes based on the Wild sum expansion.

\subsubsection{Time relaxed schemes}

Consider the differential system

$$
\partial_{t} f=\frac{1}{\varepsilon}[P(f, f)-\mu f]
$$

with initial condition $f(v, 0)=f_{0}(v)$, where $\mu>0$ is a constant and $P$ a bilinear operator. It is possible to show that the function $f$ satisfies the following formal expansion (Wild sum)

$$
f(v, t)=e^{-\mu t / \varepsilon} \sum_{k=0}^{\infty}\left(1-e^{-\mu t / \varepsilon}\right)^{k} f_{k}(v)
$$

The functions $f_{k}$ are given by the recurrence formula for $k=0,1, \ldots$

$$
f_{k+1}(v)=\frac{1}{k+1} \sum_{h=0}^{k} \frac{1}{\mu} P\left(f_{h}, f_{k-h}\right)
$$

In the case of the Boltzmann equation, that is $P(f, f)=Q(f, f)+\mu f$ formally we have that

$$
\lim _{k \rightarrow \infty} f_{k}(v)=\lim _{t \rightarrow \infty} f(v, t)=M(v)
$$

where $M(v)$ is the local Maxwellian equilibrium.

Using this remark, in [27], the following class of numerical schemes, based on a Maxwellian truncation for $m \geq 1$ of $(72)$, has been constructed

$$
f^{n+1}(v)=e^{-\mu \Delta t / \varepsilon} \sum_{k=0}^{m}\left(1-e^{-\mu \Delta t / \varepsilon}\right)^{k} f_{k}^{n}(v)+\left(1-e^{-\mu \Delta t / \varepsilon}\right)^{m+1} M(v),
$$

where $f^{n}=f(n \Delta t)$ and $\Delta t$ is a small time interval. It can be shown that the schemes obtained in this way are of order $m$ in time.

Furthermore, we have [27] the following properties for $P(f, f)=Q(f, f)+\mu f$.

\section{Proposition 1.}

(i) Conservations:

$$
\int_{\mathbb{R}^{3}} P(f, f) \phi(v) d v=\mu \int_{\mathbb{R}^{3}} f \phi(v) d v
$$

and

$$
\int_{\mathbb{R}^{3}} f^{n+1} \phi(v) d v=\int_{\mathbb{R}^{3}} f^{n} \phi(v) d v,
$$

for $\quad \phi(v)=1, v,|v|^{2}$. 
(ii) Asymptotic preservation (AP):

For any $m \geq 1$, we have

\section{Remark 7.}

$$
\lim _{\mu \Delta t / \varepsilon \rightarrow \infty} f^{n+1}=M(v)
$$

- If $B_{\Sigma}\left(\left|v-v_{*}\right|, \omega\right) \leq \Sigma$ is a bounded kernel then we can take $\mu=4 \pi \rho \Sigma$ since

$$
L_{\Sigma}[f](v):=\int_{\mathbb{R}^{3}} \int_{S^{2}} B_{\Sigma}\left(\left|v-v_{*}\right|, \omega\right) f\left(v_{*}\right) d \omega d v_{*} \leq 4 \pi \rho \Sigma
$$

Under this cut-off assumption, which is numerically essential, the solution provided by TR schemes is also nonnegative.

- We point out that for $m=0$ TR schemes are equivalent to the exact solution in a time step $\Delta t$ of the space homogeneous BGK approximation of the Boltzmann equation.

\subsubsection{Generalized Time Relaxed schemes}

The method described can be generalized by using different weight functions, with the goal of finding a best approximation for the highest order coefficients of Wild sum expansion (72).

In general this schemes can be written in the form

$$
f^{n+1}(v)=\sum_{k=0}^{m} A_{k}(t) f_{k}(v)+A_{m+1}(t) M(v),
$$

where the coefficient $f_{k}(v)$ are the determined by (73).

Let us set $\tau=1-\exp (-\mu \Delta t / \varepsilon)$, then the weights $A_{k}=A_{k}(\tau)$ are nonnegative functions which satisfy

(i) Consistency:

$$
\lim _{\tau \rightarrow 0} A_{1}(\tau) / \tau=1, \quad \lim _{\tau \rightarrow 0} A_{k}(\tau) / \tau=0, \quad k=2, \ldots, m+1
$$

(ii) Conservations:

$$
\sum_{k=0}^{m+1} A_{k}(\tau)=1, \quad \tau \in[0,1]
$$

(iii) Asymptotic preservation (AP):

$$
\lim _{\tau \rightarrow 1} A_{k}(\tau)=0, \quad k=0, \ldots, m .
$$

A possible choice of functions satisfying the above conditions are

$$
A_{k}(\tau)=(1-\tau) \tau^{k}, k=0, \ldots, m, A_{m+1}(\tau)=\tau^{m+1},
$$

which correspond to the scheme (74).

A better choice of parameters has been presented in [54]

$$
\begin{aligned}
& A_{k}(\tau)=(1-\tau) \tau^{k}, k=0, \ldots, m-1, \\
& A_{m}(\tau)=1-\sum_{k=0}^{m} A_{k}-A_{m+1}, \quad A_{m+1}=\tau^{m+2},
\end{aligned}
$$

that correspond to take $f_{m+1}=f_{m}, f_{k}=M, k \geq m+2$ in (72).

We emphasize that other choices of parameters are possible, and that the individuation of the optimal ones is an open problem. Quite remarkably the problem can be solved for Maxwellian molecules. Explicit expressions 
of the weight for the generalized first order TRMC scheme for a simple one-dimensional Maxwell model (Kac equation) have been obtained recently in [12] and read

$$
A_{0}(t)=e^{-\mu \Delta t / \varepsilon}, A_{1}(t)=\frac{4}{3}\left(e^{-\mu \Delta t /(4 \varepsilon)}-e^{-\mu \Delta t / \varepsilon}\right), A_{2}(t)=1+\frac{1}{3}\left(e^{-\mu \Delta t / \varepsilon}-4 e^{-\mu \Delta t /(4 \varepsilon)}\right) .
$$

\subsubsection{The Hybrid Time Relaxed Method}

We consider the generalized first order TR scheme for the approximation of the relaxation step (69) in the time interval $[0, t]$

$$
f^{r}(x, v, t)=A_{0}(t) f^{r}(x, v, 0)+A_{1}(t) f_{1}^{r}(x, v, 0)+A_{2}(t) M\left(f^{r}\right)(x, v, 0)
$$

where

$$
f_{1}^{r}(x, v, 0)=\frac{P\left(f^{r}, f^{r}\right)(x, v, 0)}{\mu} .
$$

Due to the structure of the operator $P(f, f)$ the extension of the componentwise hybrid method is extremely difficult. For such reason we restrict only to the case of the standard hybrid representation

$$
f(x, v, t)=(1-\beta(x, t)) f_{p}(x, v, t)+\beta(x, t) M(f)(x, v, t) .
$$

By substituting this expression at $t=0$ in the first order TR scheme (86) we obtain

$$
\begin{aligned}
f^{r}(x, v, t) & =\left(1-\beta^{r}(x, t)\right) f_{p}^{r}(x, v, t)+\beta^{r}(x, t) M\left(f^{r}\right)(x, v, t) \\
& =A_{0}(t)\left[(1-\beta(x, 0)) f_{p}(x, v, 0)+\beta(x, 0) M(f)(x, v, 0)\right] \\
& +\frac{A_{1}(t)}{\mu}\left[(1-\beta(x, 0))^{2} P\left(f_{p}, f_{p}\right)(x, v, 0)+2 \beta(x, 0)(1-\beta(x, 0)) P\left(f_{p}, M\right)(x, v, 0)\right. \\
& \left.+\beta(x, 0)^{2} \mu M(x, v, 0)\right]+A_{2}(t) M(f)(x, v, 0) .
\end{aligned}
$$

In the above equations we used the identity $P(M, M)=\mu M$. Since $M\left(f^{r}\right)(x, v, t)=M(f)(x, v, 0)$, by equating the equilibrium terms and the non equilibrium ones we obtain the evolution equations for $f_{p}^{r}(x, v, t)$ and $\beta^{r}(x, t)$. More precisely

$$
f_{p}^{r}(x, v, t)=p_{1} f_{p}(x, v, 0)+p_{2}\left[q_{1} \frac{P\left(f_{p}, f_{p}\right)(x, v, 0)}{\mu}+q_{2} \frac{P\left(f_{p}, M\right)(x, v, 0)}{\mu}\right]
$$

where

$$
\begin{aligned}
p_{1}=\frac{A_{0}(t)}{A_{0}(t)+A_{1}(t)(1+\beta(x, 0))}, & p_{2}=\frac{A_{1}(t)(1+\beta(x, 0))}{A_{0}(t)+A_{1}(t)(1+\beta(x, 0))}, \\
q_{1}=\frac{1-\beta(x, 0)}{1+\beta(x, 0)}, & q_{2}=\frac{2 \beta(x, 0)}{1+\beta(x, 0)},
\end{aligned}
$$

and

$$
\beta^{r}(x, t)=A_{0}(t) \beta(x, 0)+A_{1}(t) \beta(x, 0)^{2}+A_{2}(t) .
$$

As expected equation (90) is such that $\beta^{r}(x, t) \rightarrow 1$ as $\varepsilon \rightarrow 0$ thanks to (80)-(81).

An important feature of the TR scheme is that it maintains the hybrid representation at the next time level. The new additional difficulty is represented by the computation of the particles dynamic given by (87). To this aim note that $p_{1}, p_{2} \geq 0, p_{1}+p_{2}=1$, and $q_{1}, q_{2} \geq 0, q_{1}+q_{2}=1$, so we have a clear probabilistic viewpoint of (87) since

$$
f_{1}^{p}:=\frac{P\left(f_{p}, f_{p}\right)(x, v, 0)}{\mu}, \quad M_{1}^{p}:=\frac{P\left(f_{p}, M\right)(x, v, 0)}{\mu}
$$

are both probability densities if $f_{p}$ and $M$ are probability densities. Thus, using again a onedimensional setting with cell averages, to pick up samples $\nu_{k}$ from $f_{p}^{r}(x, v, t)$ we proceed as follows

Algorithm 6.2 (Hybrid Monte Carlo for TR schemes).

(1) Given $\rho_{j+1 / 2}(t)=\int_{\mathbb{R}^{d}} f_{j+1 / 2}(v, t) d v$ and $m=\frac{\Delta x}{N} \sum_{j} \rho_{j+1 / 2}(t)$

(2) compute $A_{0}(t), A_{1}(t), A_{2}(t)$ 
(3) for each interval $I_{j}, j=\ldots,-2,-1,0,1,2, \ldots$

(a) set $N_{j}=$ Iround $\left(\left(1-A_{2}(t)\right) \frac{\Delta x}{m} \rho_{j+1 / 2}(t)\right)$.

(b) for $k=1, \ldots, N_{j}$

compute $p_{1}, q_{1}$ according to (88)-(89)

with probability $p_{1}$ take $\left(\chi_{k}, \nu_{k}\right)$ as one of the particles in $f_{p, j+1 / 2}(v, 0)$.

with probability $1-p_{1}$ do the following

(i) with probability $q_{1}$ sample $\nu_{k}$ from $f_{1}^{p}$

(ii) with probability $1-q_{1}$ sample $\nu_{k}$ from $M_{1}^{p}$

(iii) take $\chi_{k}$ uniformly distributed in $I_{j}$

Here we omit the details on how to sample from $f_{1}^{p}$ and $M_{1}^{p}$ which is a typical feature of Direct Simulation Monte Carlo (DSMC) methods for the Boltzmann equation and are based on a fictitious collisions dynamic which allows to transform samples from $f_{p}$ into samples from $f_{1}^{p}$, and samples from $f_{p}$ and $M$ into samples from $M_{1}^{p}$ (we refer the reader to $[51,55]$ for further details).

After replacing $f_{p}$ with $f_{p}^{c}$, the above algorithms can be used directly after convection with minor modifications. In fact, convection, exactly as for the BGK model, requires a new representation step of the hybrid solution. From

$$
f^{c}(x, v, t)=\left(1-\beta^{r}(x-v t, t)\right) f_{p}^{r}(x-v t, v, t)+\beta^{r}(x-v t, t) M\left(f^{r}\right)(x-v t, v, t),
$$

we define

$$
f_{p}^{*}(x, v, t)=\left(1-\beta^{r}(x-v t, t)\right) f_{p}^{r}(x-v t, v, t), \quad \tilde{M}(x, v, t)=\beta^{r}(x-v t, t) M\left(f^{r}\right)(x-v t, v, t) .
$$

We then apply (64) and set

$$
\beta^{c}(x, t)=\min _{v} w^{c}(x, v, t), \quad M^{*}(x, v, t)=\tilde{M}(x, v, t)-\beta^{c}(x, t) M\left(f^{c}\right)(x, v, t) .
$$

Assuming $\beta^{c}(x, t) \neq 1$ we have

$$
f_{p}^{c}(x, v, t)=\frac{1}{1-\beta^{c}(x, t)}\left(f_{p}^{*}(x, v, t)+M^{*}(x, v, t)\right)
$$

Thus each time that we must take a sample from $f_{p}^{c}(x, v, t)$ in cell $I_{j}$ we can follow the strategy below.

Algorithm 6.3 (Particles sampling after convection).

(1) set $P_{j}=\frac{\rho_{p, j+1 / 2}^{*}(t)}{\rho_{p, j+1 / 2}^{*}(t)+\rho_{M, j+1 / 2}^{*}(t)}$ with $\rho_{p, j+1 / 2}^{*}(t)=\int_{\mathbb{R}^{d}} f_{p, j+1 / 2}^{*}(v, t) d v$ and $\rho_{M, j+1 / 2}^{*}(t)=\int_{\mathbb{R}^{d}} M_{j+1 / 2}^{*}(v, t) d v$.

(2) for $k=1, \ldots, N_{j}$ with probability $P_{j}$ take $\left(\chi_{k}, \nu_{k}\right)$ as one of the advected particles in $f_{p, j+1 / 2}^{*}(v, t)$. with probability $1-P_{j}$

(a) take one sample $\nu_{k}$ from the deterministic fraction $M_{j+1 / 2}^{*}(v, t)$.

(b) take $\chi_{k}$ uniformly distributed in cell $I_{j}$

\section{Remark 8.}

- One of the most important features of the hybrid method for the Boltzmann equation is that the multi-dimensional collision integral $Q(f, f)$ is always evaluated by fast Monte Carlo approaches. This is achieved thanks to the standard hybrid representation of the solution where the equilibrium function is in the kernel of $Q(f, f)$.

- The above hybrid decomposition corresponds to

$$
f(v)=\tilde{f}(v)+f_{E}(v),
$$

where now $f_{E}(v)=\beta M(v)$. Without splitting this corresponds to solve in two separate steps

$$
\partial_{t} \tilde{f}+v \nabla_{x} \tilde{f}=-\frac{1}{\varepsilon}\left(Q(\tilde{f}, \tilde{f})+2 Q\left(\tilde{f}, f_{E}\right)\right)
$$

by particles and, using the fact that $Q(\beta M, \beta M)=0$,

$$
\partial_{t} f_{E}+v \nabla_{x} f_{E}=0
$$



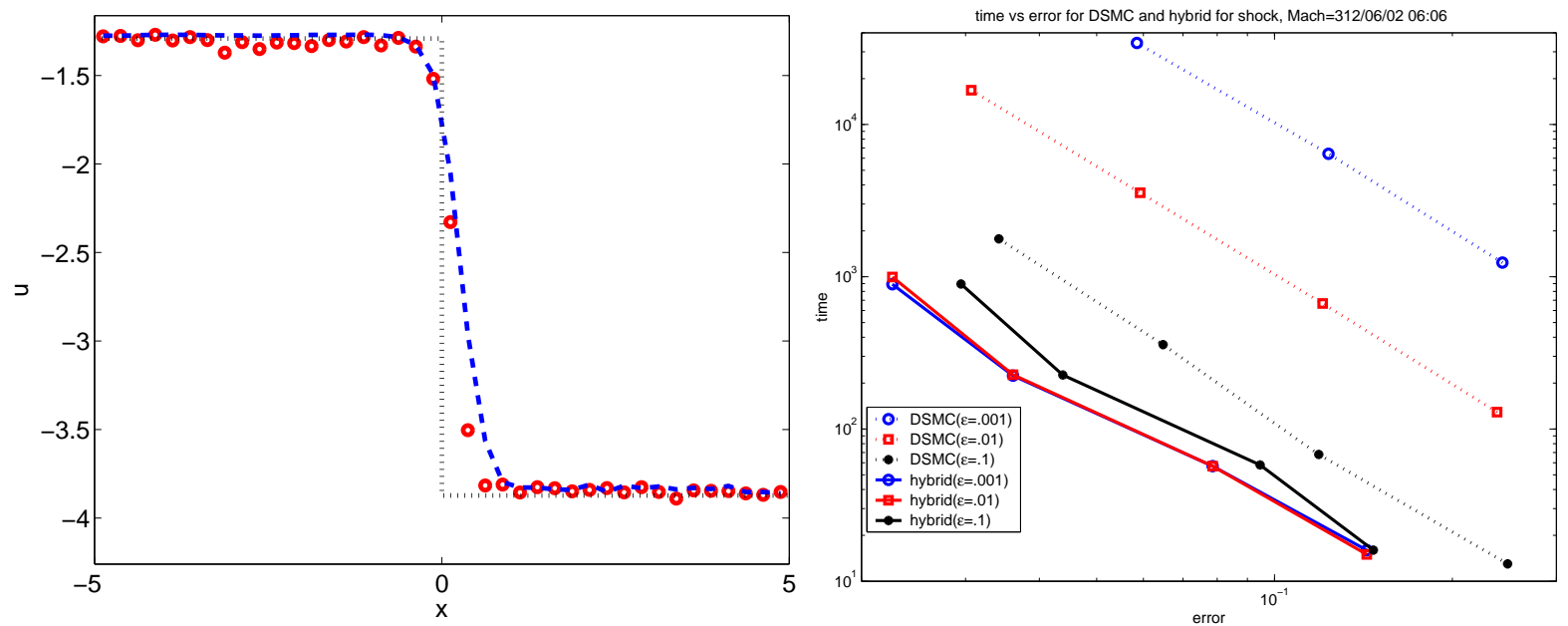

Figure 7. 1D shock profile: Mass $\rho$ for $\varepsilon=0.1$ (left) and L2-error in time for mass $\rho$ for $\varepsilon=0.1,0.01,0.001$ (right). DSMC (dots) vs hybrid method (dashed line).

with a deterministic solver. After each time step the solution need to be projected back to the form $f(v)=$ $\tilde{f}(v)+f_{E}(v)$ using a suitable representation step. Similar decompositions have been recently proposed in [45].

\subsection{Numerical results}

In this section we present some results for the hybrid method applied to the Boltzmann equation. The deterministic part of the solution here is solved using the method proposed in [26].

\subsubsection{Stationary shock wave}

The simplest problem we consider is the stationary planar shock wave (it suffices to consider shock waves with zero shock speed, since any shock can be transformed to a stationary shock by a Galilean transformation). The shock wave problem for the Boltzmann equation is solved on a finite domain $-L<x<L$ with boundary conditions that the incoming flux of particles at $x=L$ is distributed according to the Maxwellian flux $v M^{ \pm}(v)$. As initial data, we take $f(x, v, 0)=M(\rho, u, T)$, with

$$
\rho=1.0, \quad T=1.0, \quad M a=3.0, \quad L>x>0,
$$

where $M a$ is the Mach number. The mean velocity is

$$
\left.u_{x}=-M a \sqrt{(} \gamma T\right), \quad u_{y}=0,
$$

with $\gamma=5 / 3$.

The values for $\rho, u$ and $T$ for $x<0$ are given by the Rankine-Hugoniot conditions [70].

The results of the hybrid method are compared with the classical DSMC method. The result is shown in Figure 7 (left) for a Knudsen number $\varepsilon=0.1$. The number of particles in the hybrid and DSMC methods have been chosen so that the computational times are approximately equal for the two methods. The reduction of statistical error in the hybrid method is evident.

Figure 7 (right) shows a comparison of the performance of DSMC and the hybrid method, for $\varepsilon=0.1,0.01,0.001$. In this figure the computational time is plotted as a function of the statistical error for the two method. Accuracy is measured as the square root of the variance of the profile taken at 10 different times in the computation.

\subsubsection{D Flow over a Leading Edge}

Next we consider the problem of a flow over a horizontal flat plate [3]. The spatial geometry consists of a semi-infinite flat plate of zero thickness that is parallel to the incoming flow. The incoming (upstream) flow is Maxwellian with density $\rho_{0}=0.95$, velocity $u=(0,-5.16)$ and temperature $T_{0}=0.95$. The temperature of the plate is $T_{1}=1.6$. The Knudsen number is $\varepsilon=0.019$. Results for this problems are presented in Figure 8. 


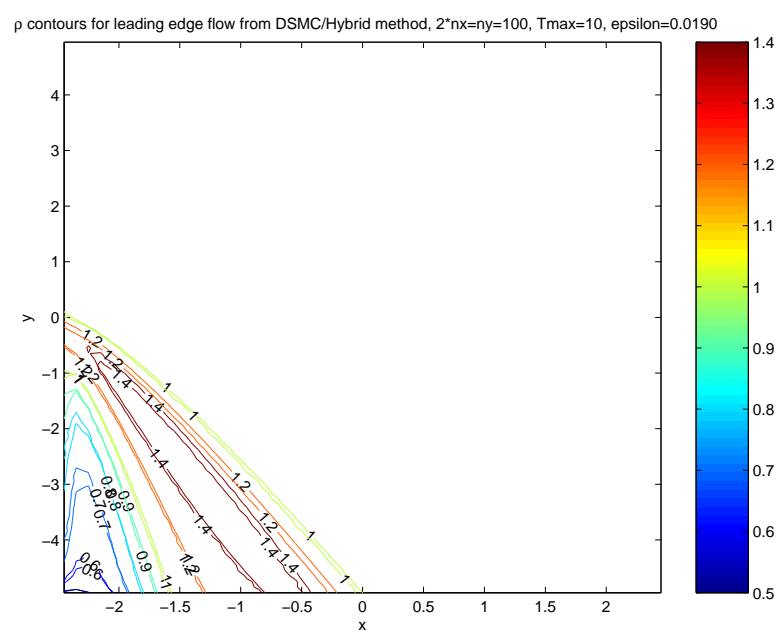

Figure 8. 2D leading edge flow: Contour plots of density for the leading edge flow problem.

\section{Concluding Remarks}

In this work we have presented several different approaches to the construction of heterogenous multiscale methods based on coupling Monte Carlo methods for the microscopic scales with finite difference/volume methods for the macroscopic scales. Applications to hyperbolic relaxation systems and kinetic equations have been discussed extensively. The hybrid schemes we developed are based on a macroscopic solver which is either a relaxation scheme or a kinetic scheme for the limit equations. Extensions of the present approach to include more general macroscopic solvers are actually under investigation.

Acknowledgements. The author would like to thank Russ Caflisch for the many stimulating discussions and for kindly providing the numerical results of section 6.3 .

\section{REFERENCES}

[1] O. Aktas, And N.R. Aluru, A combined continuum/DSMC technique for multiscale analysis of microfluidic filters. J. Comp. Phys., vol.178, (2002) pp. 342-72.

[2] H. BABovsky, On a simulation scheme for the Boltzmann equation, Math. Methods Appl. Sci., 8 (1986), pp. $223-233$.

[3] G. A. Bird, G.A.Bird Molecular gas dynamics and direct simulation of gas flows, Clarendon Press, Oxford (1994).

[4] J. F. Bourgat, P. LeTallec, B. Perthame, And Y. Qiu, Coupling Boltzmann and Euler equations without overlapping, in Domain Decomposition Methods in Science and Engineering, Contemp. Math. 157, AMS, Providence, RI, (1994), pp. 377-398.

[5] J. F. Bourgat, P. LeTallec, M.D. Tidriri, Coupling Boltzmann and Navier-Stokes equations by friction. J. Comput. Phys. 127, 2, (1996), pag. 227-245.

[6] E. Boxerman and U. Ascher, Decomposing Cloth, to appear at the ACM SIGGRAPH/EUROGRAPHICS Symposium on Computer Animation (2004).

[7] M. Briani, R. Natalini, G. Russo, Implicit-Explicit Numerical Schemes for Jump-Diffusion Processes IAC Report N.38 $(4 / 2004)$.

[8] R. E. Caflisch, Monte Carlo and Quasi-Monte Carlo Methods, Acta Numerica (1998) pp. 1-49.

[9] R. E. CAflisch, S. Jin, AND G. Russo, Uniformly accurate schemes for hyperbolic systems with relaxation, SIAM J. Numer. Anal., 34 (1997), pp. 246-281.

[10] R. E. Caflisch, and E. Luo, A Uniform Hybrid Monte Carlo Method for Simulation of Rarefied Gas Dynamics, preprint (2004).

[11] E. A. Carlen, M. C. Carvalho, and E. Gabetta, Central limit theorem for Maxwellian molecules and truncation of the Wild expansion, Comm. Pure Appl. Math., 53 (2000), pp. 370-397.

[12] E. A. Carlen, F. Salvarani, On the optimal choice of coefficients in a truncated Wild sum and approximate solutions for the Kac equation, J. Statist. Phys. 109 (2002), no. 1-2, pp. 261-277.

[13] C. Cercignani, The Boltzmann Equation and Its Applications, Springer-Verlag, New York, (1988). 
[14] C. Cercignani, R. Illner, M. Pulvirenti, The mathematical theory of dilute gases. Springer-Verlag, New York, (1995).

[15] F. Chalub, P.A. Markowich, B. Perthame, C. Schmeiser, Kinetic models for chemotaxis and their drift-diffusion limits Monatsh. Math. 142 (2004), pp. 123-141.

[16] G. Q. Chen, D. Levermore, And T. P. LiU, Hyperbolic conservation laws with stiff relaxation terms and entropy, Comm. Pure Appl. Math., 47 (1994), pp. 787-830.

[17] F. Coron, B. Perthame Numerical passage from kinetic to fluid equations. SIAM J. Numer. Anal., 28, (1991), pp. 26-42.

[18] N.Crouseilles, P.Degond, M.Lemou, A hybrid kinetic-fluid model for solving the gas-dynamics Boltzmann BGK equation, preprint (2004).

[19] S. Deshpande, A second order accurate kinetic theory based method for inviscid compressible flow. Journal of Computational Physics, (1979).

[20] G. Dimarco and L. Pareschi, Hybrid multiscale methods I. Hyperbolic systems with relaxation, preprint (2005).

[21] G. Dimarco and L. Pareschi, Hybrid multiscale methods II. Kinetic equations, work in progress (2005).

[22] W. E And B. Engquist, The heterogeneous multiscale methods, Comm. Math. Sci., 1, (2003), pp. 87-133.

[23] W. E AND B. EngQuist, The heterogeneous multiscale method for homogenization problems, SIAM J. Multiscale Modeling and Simulations (to appear).

[24] W. E And B. Engquist, Multiscale Modeling and Computation, Notices of the AMS, 50(9), (2003), pp. 1062-1070.

[25] F. Filbet, E. SonnendrüCker and P. Bertrand, Conservative Numerical schemes for the Vlasov equation. J. Comput. Phys. 172, (2001) pp. 166-187.

[26] J.A. Fleck JR., J.D. Cummings, An implicit Monte Carlo scheme for calculating time and frequency dependent nonlinear radiation transport. Journal of Computational Physics, 8, (1971), pp. 313-342, .

[27] E. Gabetta, L. Pareschi, And G. Toscani, Relaxation schemes for nonlinear kinetic equations, SIAM J. Numer. Anal., 34 (1997), pp. 2168-2194.

[28] E. Hairer And G. Wanner, Solving Ordinary Differential Equations II: Stiff and Differential-Algebraic Problems, SpringerVerlag, New York, (1987).

[29] D.B. Hash And H.A. Hassan, Assessment of schemes for coupling Monte Carlo and Navier-Stokes solution methods J. Thermophys. Heat Transf., 10, (1996), pp. 242-249.

[30] R.W. Hockney, J.W. Eastwood, Computer simulation using particles, Mc-Graw Hill International Book Co., (1981).

[31] S. Jin, Runge-Kutta methods for hyperbolic conservation laws with stiff relaxation terms, J. Comput. Phys., 122 (1995), pp. 51-67.

[32] S. Jin, C. D. Levermore, Numerical Schemes for hyperbolic conservation laws with stiff relaxation terms, J. Comp. Physics, 126 (1996), pp. 449-467.

[33] S. Jin, AND L. PARESCHI, Discretization of the multiscale semiconductor Boltzmann equation by diffusive relaxation schemes, J. Comp. Phys., 161, (2000), pp. 312-330.

[34] S. Jin, L. PARESChI, AND G. TOSCANI, Diffusive relaxation schemes for multiscale discrete-velocity kinetic equations, SIAM J. Numer. Anal., 35 (1998), pp. 2405-2439.

[35] S. Jin, L. PARESCHI, AND G. TOSCANI, Uniformly accurate diffusive relaxation schemes for multiscale transport equations, SIAM J. Numer. Anal., 35 (1998), pp. 2405-2439.

[36] S. Jin AND Z. P. XIN, Relaxation schemes for systems of conservation laws in arbitrary space dimensions, Comm. Pure Appl. Math., 48 (1995), pp. 235-276.

[37] M.H. Kalos, P.A. Whitlock, Monte Carlo Methods, Volume I: Basics, John Wiley \& Sons, New York, (1986).

[38] M.A. Katsoulakis, A.J. Majda And A. Sopasakis, Multiscale Couplings In Prototype Hybrid Deterministic/Stochastic Systems: Part I, Deterministic Closures, Comm. Math. Sci., Vol. 2, No. 2, (2004), pp. 255-294

[39] D. E. Knuth, The art of computer programming, Addison-Wesley Publ. Company, (1973)-(1981).

[40] P. LeTallec And F. Mallinger, Coupling Boltzmann and Navier-Stokes by half fluxes Journal of Computational Physics, 136, (1997) pp. 51-67.

[41] R. J. LeVeque, Numerical Methods for Conservation Laws, Lectures in Mathematics, Birkhauser Verlag, Basel (1992).

[42] Z.-H. Li AND H.-X. ZhANG, Numerical investigation from rarefied flow to continuum by solving the Boltzmann model equation International Journal for Numerical Methods in Fluids Volume, 42, 4, (2003) pp. 361-382.

[43] S. LiU, Monte Carlo strategies in scientific computing, Springer, (2004).

[44] T.-P. LiU, Hyperbolic conservation laws with relaxation, Commun. Math. Phys., 108 (1987), pp. $153-175$.

[45] T.-P. LiU And S.-H. Yu, Boltzmann Equation: Micro-Macro Decompositions and Positivity of Shock Profiles, Commun. Math. Phys. 246, (2004) pp. 133-179.

[46] J.C. Mandal, And S.M. DeshPande, Kinetic flux vector splitting for Euler equations, Computers \& Fluids, vol.23, (1994), pp. $447-78$.

[47] G. NAldi AND L. PAREschi, Numerical schemes for hyperbolic systems of conservation laws with stiff diffusive relaxation, SIAM J. Numer. Anal., 37 (2000), pp. 1246-1270.

[48] K. NanBu, Direct simulation scheme derived from the Boltzmann equation, J. Phys. Soc. Japan, 49 (1980), pp. $2042-2049$.

[49] R. NAtAlini, Convergence to equilibrium for relaxation approximations of conservation laws. Comm. Pure Appl. Math. 49 (1996) pp. 795-823 
[50] L. PARESCHI, Central differencing based numerical schemes for hyperbolic conservation laws with stiff relaxation terms, SIAM J. Num. Anal., 39, (2001), pp. 1395-1417.

[51] L. PARESChi AND R. E. CAFlisch, Implicit Monte Carlo methods for rarefied gas dynamics I: The space homogeneous case, J. Comput. Phys., 154 (1999), pp. 90-116.

[52] L. Pareschi And R. E. Caflisch, Towards an hybrid method for rarefied gas dynamics, IMA Vol. App. Math. 135, (2004), pp. 57-73.

[53] L. Pareschi, And G. Russo, Time Relaxed Monte Carlo methods for the Boltzmann equation, SIAM J. Sci. Comput. 23 (2001), pp. 1253-1273.

[54] L. Pareschi, And G. Russo, Asymptotic preserving Monte Carlo methods for the Boltzmann equation, Transport Theory Statist. Phys., 29 (2000), pp. 415-430.

[55] L. Pareschi, And G. Russo, An Introduction to Monte Carlo Methods for the Boltzmann Equation, Esaim Proceedings, Vol.10, EDP Sciences, SMAI (1999), pp. 1-38.

[56] L. Pareschi, And M.Seaid, A New Monte Carlo Approach for Conservation Laws and Relaxation Systems, Lecture Notes in Computer Science, Vol. 3037, pp. 281-288, (2004).

[57] L. PAREschi, And S. Trazzi, Numerical solution of the Boltzmann equation by Time Relaxed Monte Carlo (TRMC) methods, Int. J. Num. Meth. Fluids, (to appear).

[58] L. Pareschi, And B. Wennberg, A recursive Monte Carlo method for the Boltzmann equation in the Maxwellian case, Monte Carlo Methods Appl., 7 (2001), pp. 349-358.

[59] B. Perthame, Boltzmann type schemes for gas dynamics and the entropy property, SIAM J. Num. Anal., vol. 27, (1990), pp. 1405.

[60] D. I. Pullin, Direct simulation methods for compressible inviscid ideal gas flow, J. Comput. Phys., 34 (1980), pp. 231-244.

[61] A. Quarteroni, A. Veneziani. Analysis of a geometrical multiscale model based on the coupling of PDE's and ODE's for Blood Flow Simulations, SIAM J. on MMS, Vol.1, No. 2, (2003), pp. 173-195.

[62] R. Roveda, D.B. Goldstein, And P.L. VArghese, Hybrid Euler/Particle Approach for Continuum/ Rarefied Flows, AIAA J. Spacecraft Rockets 35, (1998), pp. 258-265.

[63] R. Roveda, D.B. Goldstein, And P.L. Varghese, Hybrid Euler/Direct Simulation Monte Carlo Calculation of Unsteady Slit Flow, AIAA J. Spacecraft Rockets 37, (2000), pp. 753-760.

[64] G. Russo, And R. E. CAflisch, Implicit methods for kinetic equations, in Rarefied Gas Dynamics: Theory and Simulations, Progress in Aeronautics and Astronautics 159, AIAA, New York, (1992), pp. 344-352.

[65] T. Schulze, P. Smereka And W. E, Coupling kinetic Monte-Carlo and continuum models with application to epitaxial growth, J. Comput. Phys., 189, (2003), pp. 197-211.

[66] G. Strang, On the construction and the comparison of difference schemes. SIAM J. Numer. Anal., 5 (1968), pp. 506-517.

[67] S. Tiwari, AND A. KlaR, An adaptive domain decomposition procedure for Boltzmann and Euler equations. J. Comp. Appl. Math., vol.90, (1998) pp. 223-37.

[68] C. Truesdell, And R. G. Muncaster, Fundamentals of Maxwell Kinetic Theory of a Simple Monatomic Gas, Academic Press, New York, (1980).

[69] W.L.WAng, Q. Sun, AND I.D. BoyD, Towards development of a hybrid DSMC-CFD method for simulating hypersonic interacting flows, AIAA 2002-3099.

[70] G. B. Whiтнам, Linear and Nonlinear Waves, Wiley-Interscience, New York, (1974).

[71] E. WILD, On Boltzmann's equation in the kinetic theory of gases, Proc. Cambridge Philos. Soc., 47 (1951), pp. $602-609$.

[72] K. Xu, L. Martinelli, And A. Jameson, Gas-kinetic finite volume methods, flux-vector splitting, and artificial diffusion. J. Comp. Phys., vol.120, (1995) pp. 48-65 